\title{
First high-statistics and high-resolution recoil-ion data from the WITCH retardation spectrometer
}

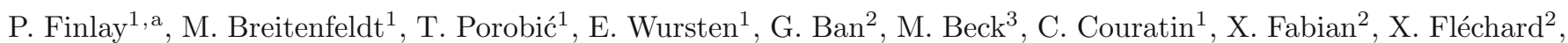
P. Friedag ${ }^{3}$, F. Glück ${ }^{4}$, A. Herlert ${ }^{5}$, A. Knecht ${ }^{1,6, b}$, V.Y. Kozlov ${ }^{4}$, E. Liénard ${ }^{2}$, G. Soti ${ }^{1}$, M. Tandecki ${ }^{7}$, E. Traykov $^{8}$, S. Van Gorp ${ }^{9}$, Ch. Weinheimer ${ }^{3}$, D. Zákoucký ${ }^{10}$, and N. Severijns ${ }^{1}$

${ }^{1}$ KU Leuven University, Instituut voor Kern-en Stralingsfysica, Celestijnenlaan 200D, 3001 Leuven, Belgium

2 Normandie Univ., ENSICAEN, UNICAEN, CNRS/IN2P3, LPC Caen, 14000 Caen, France

3 Universität Münster, Institut für Kernphysik, Wilhelm-Klemm-Strasse 9, D-48149 Münster, Germany

4 Karlsruhe Institute of Technology, Institut für Kernphysik, Postfach 3640, 76021 Karlsruhe, Germany

5 FAIR, Planckstr. 1, 64291 Darmstadt, Germany

${ }^{6}$ PH Department, CERN, CH-1211 Geneva 23, Switzerland

7 TRIUMF, 4004 Wesbrook Mall, Vancouver BC, V6T 2A3, Canada

8 GANIL, CEA/DSM-CNRS/IN2P3, Bvd Henri Becquerel, 14076 Caen, France

9 Atomic Physics Laboratory, RIKEN, Saitama 351-0198, Japan

10 Nuclear Physics Institute, ASCR, 25068 Rez, Czech Republic

Received: 3 June 2016

Published online: 27 July 2016

(C) The Author(s) 2016. This article is published with open access at Springerlink.com

Communicated by C. Broggini

\begin{abstract}
The first high-statistics and high-resolution data set for the integrated recoil-ion energy spectrum following the $\beta^{+}$decay of ${ }^{35} \mathrm{Ar}$ has been collected with the WITCH retardation spectrometer located at CERN-ISOLDE. Over 25 million recoil-ion events were recorded on a large-area multichannel plate (MCP) detector with a time-stamp precision of $2 \mathrm{~ns}$ and position resolution of $0.1 \mathrm{~mm}$ due to the newly upgraded data acquisition based on the LPC Caen FASTER protocol. The number of recoil ions was measured for more than 15 different settings of the retardation potential, complemented by dedicated background and half-life measurements. Previously unidentified systematic effects, including an energy-dependent efficiency of the main MCP and a radiation-induced time-dependent background, have been identified and incorporated into the analysis. However, further understanding and treatment of the radiation-induced background requires additional dedicated measurements and remains the current limiting factor in extracting a betaneutrino angular correlation coefficient for ${ }^{35} \mathrm{Ar}$ decay using the WITCH spectrometer.
\end{abstract}

\section{Introduction}

The Standard Model (SM) is a pillar of modern physics, successfully describing not only the experimentally observed fundamental particles [1,2], but the strong and electroweak interactions as well [3]. Despite its success, however, the SM remains an incomplete theory, with parity violation and the excess of matter over antimatter, for example, remaining unexplained. Being a parameter-based model, it is left up to experiments to place constraints on, or hopefully expose, new parameters which may shed light on some of these as yet unexplained observations. The weak interaction, for example, is prescribed as purely

\footnotetext{
a e-mail: paul.finlay@kuleuven.be

b Present address: Paul Scherrer Institute, Villigen CH-5232, Switzerland.
}

vector $(\mathrm{V})$ minus axial-vector $(\mathrm{A})$, but the most general $\beta$ decay Hamiltonian consistent with Lorentz invariance $[4$, 5] also allows for scalar (S), tensor (T), and pseudoscalar (P) type interactions. While the weak interaction has been shown experimentally to be predominantly of a V-A nature, the presence of $\mathrm{S}$ and $\mathrm{T}$ currents has not been ruled out, with the present limits (at 95\% confidence level)

$$
\left|C_{S}^{(\prime)} / C_{V}\right|<0.07 \text { and }\left|C_{T}^{(\prime)} / C_{A}\right|<0.09
$$

coming from the most recent global fit of selected experimental results in nuclear $\beta$ and neutron decay [6] (see also e.g. $[7-10])$.

It is possible to search for these exotic currents via careful study of the kinematics and correlations following $\beta$-decay and making comparisons to SM predictions. 


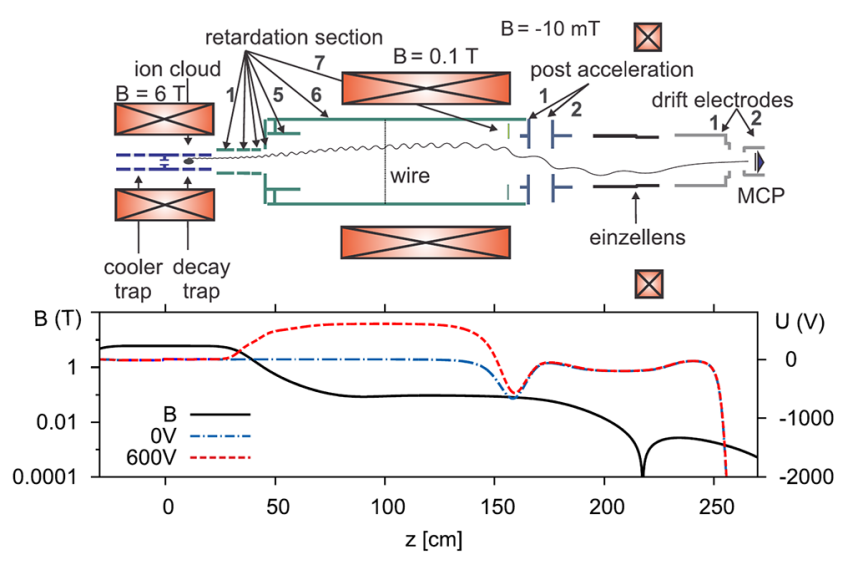

Fig. 1. (Colour online) Overview of (top) the Penning traps, retardation spectrometer, re-acceleration section, and main MCP detector and (bottom) the magnetic field strength (black line) and electric potentials with the spectrometer at $600 \mathrm{~V}$ (red dashed line) and $0 \mathrm{~V}$ (blue dot-dashed line) throughout this region. The oscillating line (top) represents a typical daughterion trajectory from the decay Penning trap to the main MCP.

In recent years atom and ion traps have been adopted in these searches [11-18], particularly for $\beta-\nu$ correlation measurements as they enable the preparation of isotopically pure, well-localized sources which are easily manipulated and have well-understood properties. The conditions present in atom and ion traps reduce the effects of scattering of the $\beta$ particles and recoil ions, which is a limitation when sources are implanted in materials. Whereas most $\beta-\nu$ correlation experiments measure coincidences between the recoil ions and the $\beta$ particles to infer the neutrino momenta, the Weak Interaction Trap for CHarged particles (WITCH) experiment [19-25], situated at CERN-ISOLDE [26], uses a double Penning trap and retardation spectrometer (fig. 1) to measure the energy spectrum of the recoiling daughter nuclei, in singles. The advantages of this approach for a $\beta-\nu$ angular correlation measurement are twofold: high statistics due to the focusing of the recoil ions towards the main detector by the magnetic field, and sensitivity to systematic effects which differ from those encountered in experiments that depend on a coincidence measurement. The latter of these characteristics is crucial in any search for new physics in order to cross-check results and establish confidence in limits imposed, or discoveries made, by other experiments.

The WITCH experiment, first described in 2003 [19, $20]$, has seen many technical developments and upgrades over its lifetime [27-31]. The first recorded recoil-ion spectrum, measured for ${ }^{124} \mathrm{In}$, was reported in 2008 [23], while the first result for the $\beta-\nu$ correlation in ${ }^{35} \mathrm{Ar}$ decay was reported in 2014 [25], albeit with limited statistics. Recent efforts have been focused on an improved measurement for the $\beta-\nu$ correlation in ${ }^{35} \mathrm{Ar}$ decay as its SM value can be calculated to better than $1 \%$, the half-life of ${ }^{35} \mathrm{Ar}$ $\left(T_{1 / 2}=1.7752(10) \mathrm{s}[32]\right)$ is in a range appropriate for this technique, and ${ }^{35} \mathrm{Ar}$ is produced in large quantities at ISOLDE.
In this paper we present the first high-statistics and high-resolution recoil-ion data obtained with the WITCH spectrometer for ${ }^{35} \mathrm{Ar}$. Several major upgrades, implemented since the result reported in ref. [25], have enabled the improvement in resolution and statistics which have ultimately led to the exposition of previously unobserved systematics associated with this measurement technique. A comprehensive analysis of the data, as well as a detailed study of these new systematic effects and their impact on the $\beta-\nu$ correlation measured with a retardation spectrometer, is presented.

\section{Experiment}

The ions of interest, ${ }^{35} \mathrm{Ar}$, were produced by $\sim 2 \mu \mathrm{A}$ of protons at $1.4 \mathrm{GeV}$ from the proton synchrotron booster (PSB) impinging on a $\mathrm{CaO}$ micro-structured target connected to a versatile arc discharge ion source (VADIS) [33]. With a cold transfer line elements other than noble gases were suppressed. Following magnetic separation the $A=$ 35 beam was delivered to the REXTRAP [34] Penning trap, where the ions were cooled via collisions with neon buffer gas before being extracted as a bunched ion beam at $30 \mathrm{kV}$. Starting with the ejection of the ion bunch from REXTRAP, the WITCH experimental cycle can be subdivided into three distinct phases: accumulation, preparation, and recoil-ion measurement. The voltage sequences associated with each phase, as well as the data from a typical recoil-ion energy measurement, are displayed in fig. 2.

\subsection{Accumulation phase}

The properties of the ion cloud following ejection from REXTRAP are not suited to a precision measurement of the recoil-ion energy. Therefore WITCH contains a dedicated cylindrical Penning trap for the accumulation and preparation of ${ }^{35} \mathrm{Ar}$ ions with the desired properties. Before reaching this so-called "cooler" Penning trap (CT) the ion bunch is steered and focused through a horizontal section of the beam line (HBL), followed by a $90^{\circ}$ bend to a vertical section of the beam line (VBL), illustrated in fig. 3. The VBL contains a pulsed drift tube (PDT), a $70 \mathrm{~cm}$ long electrode [28] which is initially biased to a potential of $21 \mathrm{kV}$. Upon entering the PDT region the ion bunch thus has a kinetic energy of $9 \mathrm{keV}$, at which point the PDT is switched within $2 \mu$ s to $-9 \mathrm{kV}$, leaving the ion bunch with a net energy of approximately $0 \mathrm{keV}$. The PDT is followed by a series of electrodes whose potentials range from $-5 \mathrm{kV}$ to $0 \mathrm{kV}$ leading up to injection of the ion bunch into the $\mathrm{CT}$, which is located in the $13 \mathrm{~cm}$ diameter bore of a $6 \mathrm{~T}$ Oxford Instruments superconducting magnet. A blocking potential of approximately $100 \mathrm{~V}$ is applied to the CT end caps in order to confine the lowenergy ions in this region, while the upstream end cap is lowered briefly to $0 \mathrm{~V}$ to allow the injection of a new ion bunch. This process of accumulation in REXTRAP followed by pulsing down the ion bunch's energy in the PDT before injection into the WITCH CT was performed three 

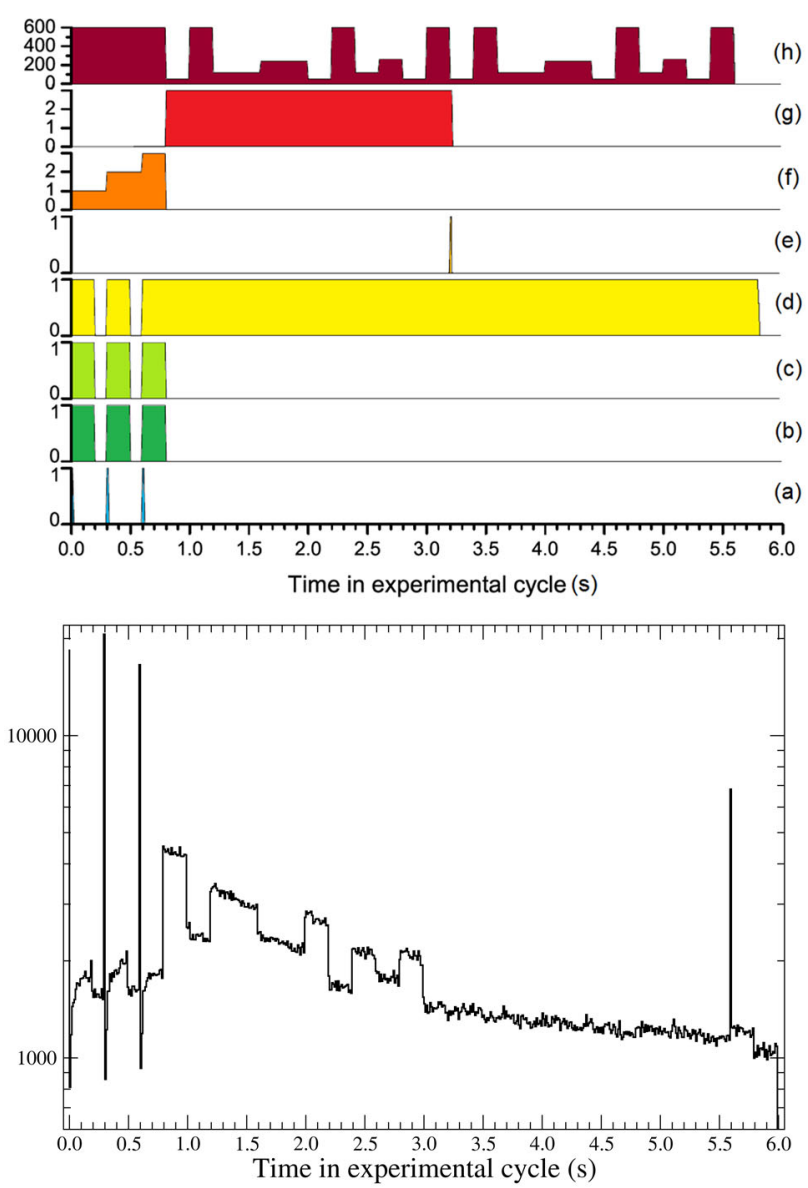

Fig. 2. (Colour online) Top: graphical depiction of the time structure for a typical recoil-ion cycle showing (a) the triggers from REXTRAP, i.e. when the ion bunches are injected into the WITCH cooler trap, (b and c) the lowering (0) and raising (1) of the voltages on the lower and upper endcaps, (d) the gate applied to one of the steerer quadruplets in the vertical beamline, with 0 indicating normal operation and 1 indicating that the element was grounded, (e) the radial ejection of the ions from the decay trap via a dipole excitation, (f) the accumulation of the ions in the cooler trap and $(\mathrm{g})$ the period when the ions are in the decay trap. A typical retardation voltage sequence (h) is also shown for comparison (vertical scale in volts). Bottom: MCP data from a typical recoil-ion run. Note that the part of the cycle following the radial ejection of the ions from the decay trap is used to measure background components.

times at the beginning of each cycle to improve statistics, necessitated by the relatively poor transmission efficiency of $1-5 \%$ that was achieved between REXTRAP and WITCH during this beam time.

\subsection{Preparation phase}

The accumulated ions' energy is further reduced during the preparation phase via collisions with He buffer gas which is being continuously flowed into the CT trap. During cooling of the ions' energy the end caps are lowered and a quadrupolar trapping potential with a depth

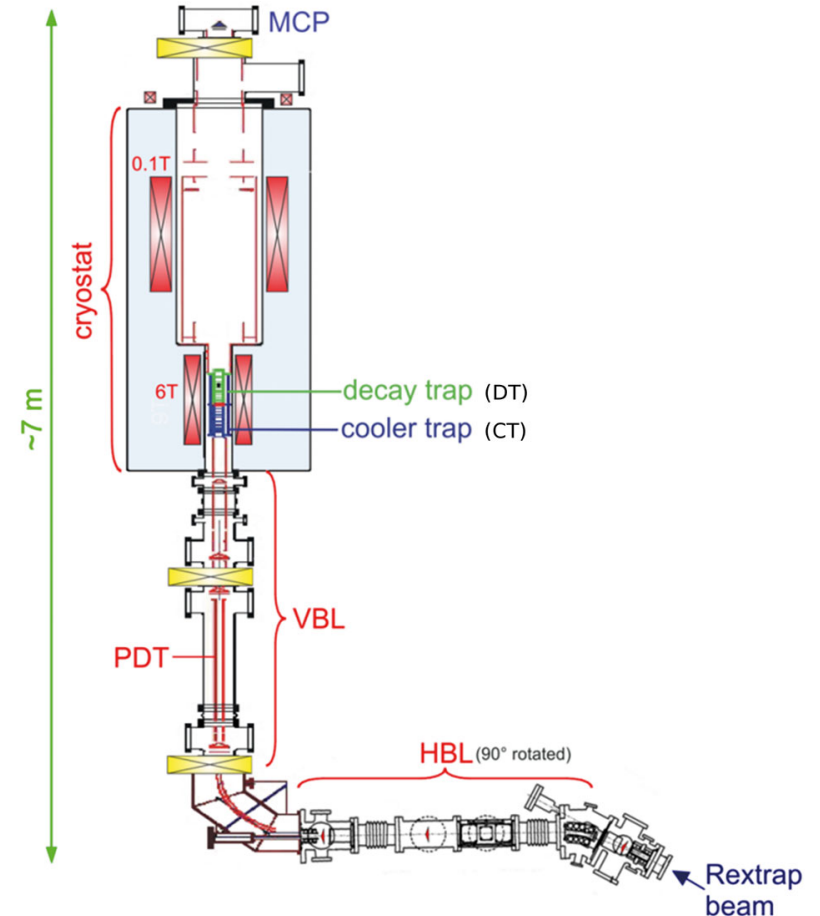

Fig. 3. (Colour online) Schematic drawing of the WITCH setup. See text for details.

of between $10 \mathrm{~V}$ and $15 \mathrm{~V}$ is applied to confine the lowenergy ion cloud in the center of the trap. As the collisional cooling process results in an increase of the cloud's magnetron radius [35], an $\mathrm{RF}$ sinusoidal excitation potential is applied at the free-particle cyclotron frequency to the central segmented ring electrode of the CT to counteract the increase in magnetron radius and facilitate further cooling and centering of the cloud. After a few $100 \mathrm{~ms}$ the cloud is sufficiently cooled and centered that it can then be transferred through a $2 \mathrm{~mm}$ diameter, $7 \mathrm{~cm}$ long pumping diaphragm to the second cylindrical Penning trap of the WITCH system, the so-called "decay" trap (DT). The purpose of the narrow pumping diaphragm is to confine the He buffer gas to the CT region and ensure the ambient pressure in the DT and retardation region is as low as possible.

\subsection{Recoil-ion measurement phase}

The ion cloud in the DT now has sufficiently low energy $(0.025 \mathrm{eV}[36])$ and localization in space to act as a scattering-free point-like radioactive source. Recoil ions which have enough energy to overcome the shallow $15 \mathrm{~V}$ trapping potential are able to escape from the decay trap and be detected. These recoil ions with trajectories towards the downstream end of the DT will enter the retardation spectrometer of the MAC-E filter type [37,38] (similar to e.g. the KATRIN [39] and aSPECT [40] experiments), where their energy is probed. The transition from the high magnetic field of $6 \mathrm{~T}$ in the trapping region to the low magnetic field of $0.1 \mathrm{~T}$ in the retardation spectrome- 
ter section causes an adiabatic transformation of the recoil ions' radial energy into axial energy with high efficiency (i.e. $\approx 98.3 \%$ ) [20], thus allowing the total recoil energy to be probed via the retardation electrode. The recoil ions' energy is not measured directly, but rather the retardation electrode is set to a potential between $0 \mathrm{~V}$ and the recoilion endpoint energy for ${ }^{35} \mathrm{Ar}$ decay of $\approx 452 \mathrm{eV}$ [25], such that the fraction of recoil ions with energy above the retardation potential are able to pass through this section, where they are post-accelerated to a few $\mathrm{keV}$ and focused onto a position-sensitive MCP detector. In this way an integrated energy spectrum is obtained by counting the ions as a function of the height of the potential barrier.

At the midpoint of the recoil-ion measurement phase of the cycle the DT is emptied by ejecting any remaining ${ }^{35} \mathrm{Ar}$ upstream while simultaneously applying a dipole excitation at the magnetron frequency to drive the ions to larger radii so they may be neutralized on the walls of the trap and subsequently pumped away. The remaining half of the cycle is then dedicated to measuring residual backgrounds in the spectrometer (see sect. 3.2.2).

\subsection{Dedicated background measurement cycle}

To further characterize the background components present in these measurements a dedicated background cycle was measured following each of the decay data cycles described so far (fig. 2). All of the steps in the cycle described in sects. 2.1, 2.2, and 2.3 were executed in the dedicated background cycle as well, however, while the ion beam was traversing the HBL in the accumulation phase an electrostatic kicker was engaged to deflect the ions to the walls of the beam line, thus preventing any radioactivity from entering the system.

In addition to the sequences described above for recoilion and dedicated background measurement cycles, several runs devoted to the measurement of the half-life of ${ }^{35} \mathrm{Ar}$ were also performed during this beam time. The unique cycle structures for the half-life runs are described in sect. 3.1.

\subsection{Technical upgrades}

Following the previous experimental campaign [25] it was decided to upgrade the existing data acquisition system, based on a MCS card and CAMAC-based analog electronics [23], to a fast digital system capable of handling a high event rate with minimal dead time. The FASTER (Fast Acquisition SysTem for nuclEar Research) system is a modular, triggerless, digital DAQ designed to fully replace standard NIM and CAMAC/VME modules used in nuclear physics applications [41]. Time and charge measurements (TDC/QDC) are executed on modular CARAS daughter cards which digitize the signal for on-board processing [42]. After digitization, the signal is timestamped, discriminated and the charge integrated, yielding time and charge information. The digitization is performed on a

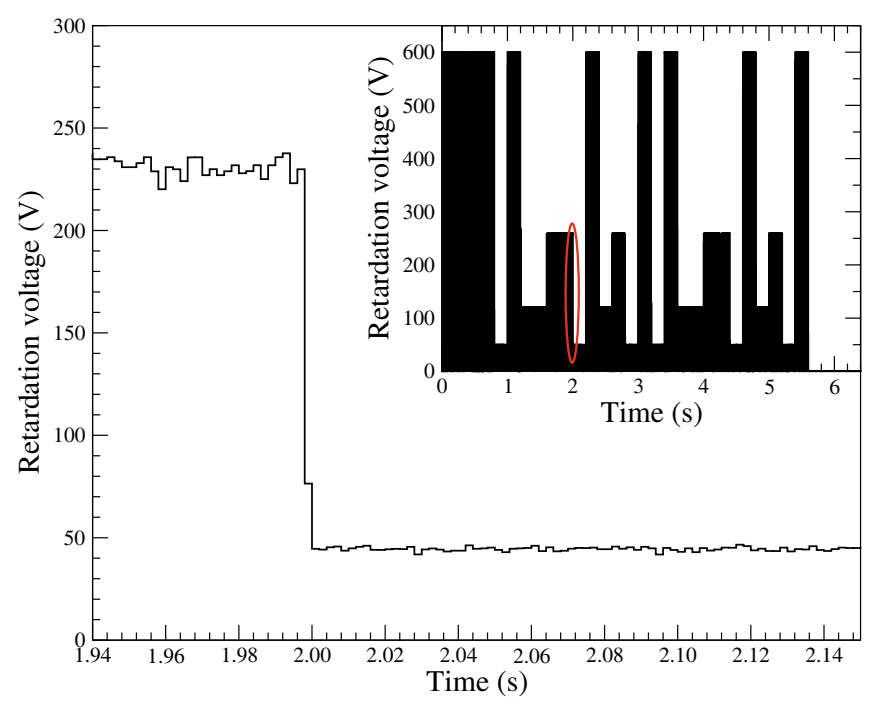

Fig. 4. The full retardation voltage sequence (inset) for a typical run, and a portion of the same sequence around $2 \mathrm{~s}$ shown with $2 \mathrm{~ms} /$ bin resolution. While the precision with which the retardation voltage is recorded is somewhat poor, the superior timing resolution of FASTER allows for precise monitoring of relative changes in the applied potential versus time. During the offline analysis this allows for the exclusion of periods in the cycle during which the retardation voltage is in transition.

12 bit $500 \mathrm{MHz}$ analog-to-digital converter, with a timestamp accuracy of $2 \mathrm{~ns}$. The particular FASTER configuration employed at WITCH consists of 8 TDC/QDC channels to accommodate the four MCP delay lines, the main MCP signal, the pulse associated with the ejection of the ion bunch from REXTRAP to time the execution of the control sequences associated with the cycle, and a signal proportional to the applied retardation voltage (fig. 4) to monitor the time evolution of the applied potential.

One immediate advantage of the low deadtime associated with FASTER is the ability, for the first time, to reconstruct the recoil data event by event. Events recorded with very high QDC values that cause the FASTER charge integration to saturate were found to be uniformly distributed in time throughout the cycle (fig. 5). These events are most likely associated with background ionization rather than true recoil events, and are thus safely rejected in the offline analysis via the FASTER QDC saturation flag.

\section{Analysis}

The TDC and QDC information for the seven FASTER channels were recorded event by event in a proprietary FASTER format, which was then converted into ROOT trees [43] for further analysis. These data were then sorted to include only events for which each of the four delay line signals was present in addition to the main MCP signal [44], and none of the QDC channels had their saturation flag set. The delay-line signals were used to reconstruct the position information for the event, while 


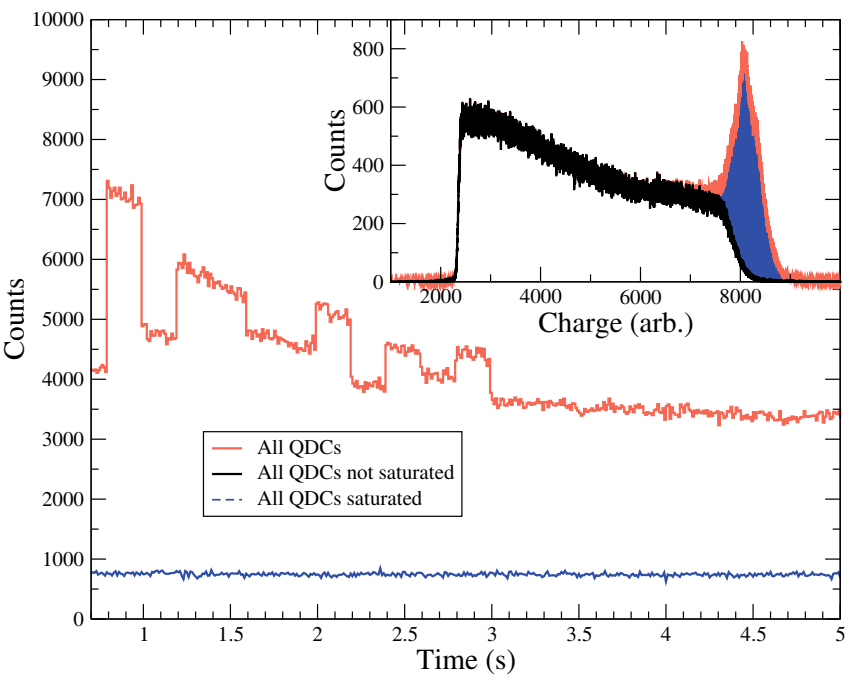

Fig. 5. (Colour online) The QDCs (inset) for all data (red), those events which do not saturate the FASTER QDC (black), and all events which do saturate the QDC (blue). The distribution of the saturation events in time is uniform, likely associated with background ionization, and thus safely rejected during the offline analysis.

the cycle-by-cycle main MCP signal versus time was extracted from the sorted ROOT files and placed into matrices for each run. Further cycle-rejection procedures were carried out on the main MCP matrices prior to fitting, as discussed in sect. 3.2.1. The fitting routine used is based on a Levenberg-Marquardt algorithm from Numerical Recipes in $C$ [45] and has e.g. been used extensively in the superallowed Fermi beta-decay program at TRIUMF (see ref. [46] and references therein).

\subsection{Dedicated half-life runs}

With a radioactive half-life of $1.7752(10) \mathrm{s}$ [32] most of the ${ }^{35} \mathrm{Ar}$ initially confined in the DT will have decayed away by the end of the $6 \mathrm{~s}$ cycle, and these losses must be precisely accounted for in order to extract the correct recoil-ion amplitudes. Unfortunately additional loss mechanisms are present for the ${ }^{35} \mathrm{Ar}$ which are particular to this experiment, negating the advantage of a precisely determined ${ }^{35} \mathrm{Ar}$ half-life. Dedicated measurements were thus performed during this beamtime to determine the effective half-lives associated with the various signals in our detector. Due to the particularities of both the apparatus and the cycle structure, the signals registered with the MCP can be characterized by three distinct half-lives.

\subsubsection{Implant half-life}

While loading the $\mathrm{CT}$ at the beginning of the cycle the downstream end cap is left at a high potential in order to prevent the ions from escaping. In principle the ions have very little kinetic energy following their passage through

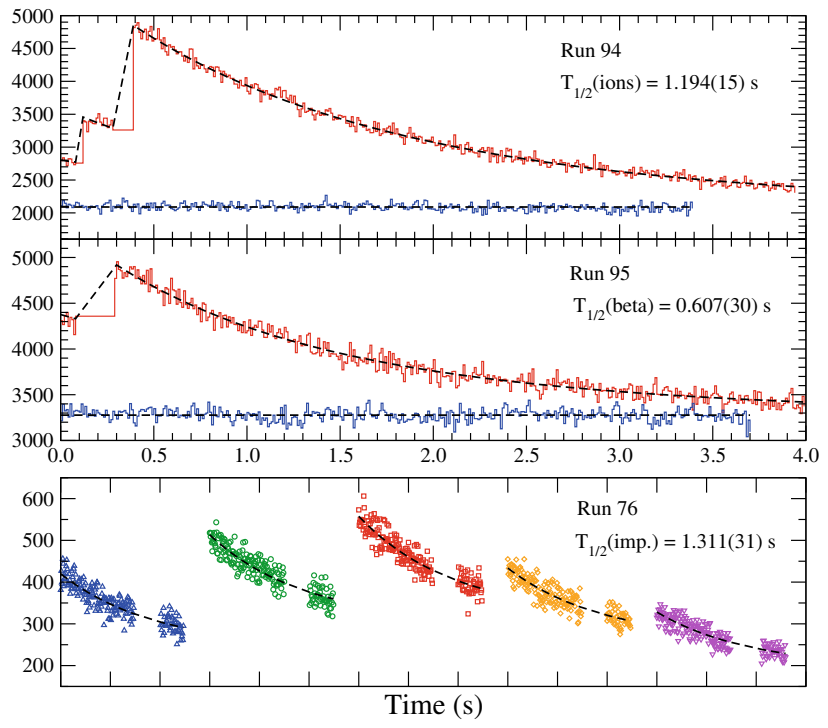

Fig. 6. (Colour online) Dedicated implant (bottom), beta/implant (middle) and ion/beta/implant (top) half-life runs with resulting fits overlaid. The five implantation decay curves are staggered horizontally to aid the eye. See text for details.

the PDT and should therefore be blocked by a modest potential, however, the fringes of the ion bunch will not be properly pulsed down in energy due to the finite size of the PDT and as a result they overshoot the trap and are deposited on the surface of the MCP. Calculations performed with SRIM [47] show that at $30 \mathrm{keV}$ the ${ }^{35} \mathrm{Ar}$ penetrates to an average depth of 28(8) nm into the surface of the MCP [48], but the actual penetration depth will be shallower as most of the ${ }^{35} \mathrm{Ar}$ ions will have had their energy at least partially reduced when passing through the PDT. This implanted activity on the MCP, present throughout the entire cycle, does not decay with the true ${ }^{35} \mathrm{Ar}$ halflife, but rather an effective half-life due to diffusion of the noble gas from the surface of the MCP. Also important to note is that this activity is downstream of the spectrometer and thus does not react to changes in the retardation potential.

In order to quantify the effect of diffusion on the measured ${ }^{35} \mathrm{Ar}$ half-life a dedicated run was performed with the trap electrode settings as they were for a typical cycle, but without pulsing down the ion bunch in the PDT before "loading" the trap, effectively depositing all the activity on the surface of the MCP. The data is presented in the lower panel of fig. 6 . There are five separate curves, each with a gap towards the end, due to the switching of the retardation voltage on the spectrometer from $600 \mathrm{~V}$ to $100 \mathrm{~V}$ during this portion of the cycle. This switching of the potential has no impact on the implanted activity, but does result in a different focusing of the background of ionized rest gas onto the MCP. Since no dedicated background run was performed for these settings, these sections of the decay data had to be omitted when fitting. To compound the issue, the retardation sequence lost synchronization with the cycle, and so the switch from $600 \mathrm{~V}$ to $100 \mathrm{~V}$ did 
not take place at exactly the same time in every cycle, but appeared at five separate times. All the cycles where the retardation voltage switched at the same time in the cycle were summed, and these five groups of implantation decay data, with fit overlaid, are presented in the bottom panel of fig. 6 .

\subsubsection{Beta half-life}

The high energy of the beta particles following the decay of the ${ }^{35} \mathrm{Ar}$ ions in the DT $\left(Q_{\beta^{+}}=5966.1(7) \mathrm{keV}\right.$ [49]) prevents them from being blocked by the spectrometer. The recent addition of a $-10 \mathrm{mT}$ air-cooled magnet (magneticfield vector opposite to those of the superconducting magnets) after the post-acceleration region of the spectrometer helps reduce the beta intensity reaching the MCP by breaking up the magnetic-field lines in this region [25], but the betas' contribution to the recorded MCP signal cannot be completely eliminated and must be accounted for in the fits.

A dedicated beta half-life run was performed by loading the traps in the usual manner for a measurement cycle, but the retardation spectrometer was left at $600 \mathrm{~V}$ in order to block all recoiling ions exiting the trap. While loading the trap, some ${ }^{35} \mathrm{Ar}$ activity will always overshoot the endcap and be implanted into the MCP surface, so the beta and implant half-lives must be fit together. The dedicated implantation half-life run presented in sect. 3.1.1 was thus used to constrain the implantation half-life. While the ions are confined to the CT trap only background and implantation activity are effectively registered by the MCP due to the small size of the pumping diaphragm's aperture, thus the $30 \mathrm{~ms}$ before the ions are transferred to the DT from the CT are used to constrain the implantation activity. These data, including fit and dedicated background measurement, are presented in the middle panel of fig. 6 .

\subsubsection{Ion half-life}

The final signal with an effective half-life to be considered is that of the recoil ions themselves. Ideally the recoil-ion activity would decay with the true ${ }^{35} \mathrm{Ar}$ half-life, but neutralization of the ions due to charge exchange with the trap walls or residual gas can free them from the confining potential of the DT and they can then be pumped away, leading to additional losses and affecting the resulting half-life. To quantify this effective half-life a dedicated run was performed with all cycle parameters the same as a typical recoil-ion run, but with the retardation voltage sequence replaced with a constant retardation potential of $50 \mathrm{~V}$. With radioactivity in the system a large count rate is observed if the spectrometer is switched below $50 \mathrm{~V}$, much larger than expected from the trapped ions alone, and is likely due to ionized rest gas which becomes trapped in a pseudo Penning trap established between the spectrometer and PDT [50]. Further discussion of such a background appears in sect. 4.2 .
Table 1. Values for the various effective half-lives of ${ }^{35}$ Ar measured at WITCH.

\begin{tabular}{llll}
\hline \hline Year & Trapped Ions (s) & Implanted Ions (s) & Betas (s) \\
\hline $2007[50]$ & - & $1.38(8)$ & - \\
$2009[50]$ & $1.10(17)$ & $1.31(6)$ & $1.12(3)^{*}$ \\
$2011[51]$ & $1.32(6)$ & - & $0.67(1)^{* *}$ \\
$2012^{\dagger}$ & $1.194(15)$ & $1.311(31)$ & $0.607(30)$ \\
$2012^{\ddagger}$ & - & $1.282(11)$ & - \\
\hline * This beta half-life was fit without regard for the underlying \\
implanted activity. \\
** This "background" half-life value was not divided into betas and \\
implanted ions. \\
$\dagger$ Obtained from fits to the dedicated half-life runs (sect. 3.1). \\
$\ddagger$ Obtained from fitting the recoil spectrum (sect. 3.2.3).
\end{tabular}

Since $50 \mathrm{~V}$ on the retardation spectrometer is insufficient to block the beta particles, their activity as well as the implantation activity need to be accounted for in this fit. The implantation activity was constrained in the same way as was done when determining the beta half-life. The beta activity was constrained during the $170 \mathrm{~ms}$ period after the ions were transferred to the decay trap but before the retardation voltage was switched from $600 \mathrm{~V}$, when no ions could pass, down to $50 \mathrm{~V}$. This final run with all three signals present, including a dedicated background measurement, is displayed with fit overlaid in the top panel of fig. 6 .

Due to the convoluted nature of the signals impinging on the MCP, these three dedicated half-life runs were fit simultaneously with a single function describing separate amplitudes for all the components in the various measurements, but three global parameters for the implant, beta, and ion half-lives, which are common to all runs. The implantation half-life of $1.311(31) \mathrm{s}$ and the recoil-ion halflife of 1.194(15)s measured during this beam time agree well with previous values measured at WITCH on similar MCPs (see table 1). An anomalously low value for the effective half-life of the "background" signal, which was composed primarily of betas, was also recorded at WITCH during a beam time in 2011 (table 1), but the reason for such a low value for the beta half-life, and why this component has a half-life different from the recoil-ion half-life, is still not fully understood. One possible explanation is that this effect could be caused by the particular motion of the ions in the trap, as the ion cloud was found to not be perfectly centered in the trap due to a misalignment of the electric and magnetic fields and thus exhibits a magnetron oscillation about the center of the trap. Since the trajectories of the beta particles and recoil ions are very different both within the trap itself and through the spectrometer, then it is possible that betas from different proportions of the cloud are transmitted to the MCP during different phases of the magnetron motion in the trap, thus looking like an additional loss mechanism for betas. Evidence for this hypothesis is discussed further in sect. 3.2.3, and the empirical value for the beta half-life measured here is adopted during the recoil-ion analysis. 
Table 2. Details of the 12 recoil-ion runs used in the analysis for extracting $a_{\beta \nu}$ in ${ }^{35} \mathrm{Ar}$ decay. Retardation potential values of $50 \mathrm{~V}$ and $600 \mathrm{~V}$ were also used in every cycle for normalization purposes in addition to the retardation potentials listed. For details on the bad-cycle rejection procedure see sect. 3.2.1.

\begin{tabular}{llll}
\hline \hline Run \# & $\begin{array}{l}\text { Total } \\
\text { cycles }\end{array}$ & Rejected cycles & $\begin{array}{l}\text { Retardation } \\
\text { potentials }(\mathrm{V})\end{array}$ \\
\hline 96 & 266 & 40 & 120,260 \\
97 & 268 & 42 & 20,200 \\
98 & 267 & 46 & 100,420 \\
99 & 268 & 18 & 80,300 \\
100 & 267 & 13 & 160,360 \\
101 & 273 & 40 & 300,220 \\
102 & 268 & 10 & 500,20 \\
103 & 268 & 31 & 340,120 \\
104 & 267 & 24 & 260,60 \\
106 & 268 & 22 & 40,340 \\
107 & 267 & 24 & 140,280 \\
108 & 268 & 22 & 460,320 \\
\hline \hline
\end{tabular}

\subsection{Recoil-ion runs}

A total of 90 recoil-ion runs comprising 25 million events were taken over the course of the experiment. However, due to relatively poor transmission efficiency, lower-thanexpected isotope production, and ionization of the rest gas in the system, the full data set is of mixed quality and contains many runs with a very poor signal-to-noise ratio and/or spontaneously emitted or wildly varying background. Thus a sequence of 12 of the highest quality runs was used to extract amplitudes for the recoil-ion intensities. These runs and their associated statistics are tabulated in table 2 .

\subsubsection{Cycle selection}

An initial event selection was done during the offline sorting of the raw data, where events within a cycle were rejected if the saturation flag of the FASTER QDC was set. The individual recoil-ion cycles comprising a run did not have a sufficient signal-to-noise ratio to fit directly for the recoil-ion amplitudes, and thus the cycles for each run had to be summed and fit. Since fluctuating background and "noise bursts" were still present, though to a lesser degree, in the 12 highest-quality runs, a cycle rejection procedure was carried out prior to fitting this data. Background cycles which did not display a constant rate were rejected, and recoil-ion cycles were kept only if the background rate was the same both immediately before and immediately after that cycle, and the recoil-ion cycle itself did not exhibit any obvious noise or anomalous behaviour such as counting rates fluctuating wildly with time (see fig. 7). The number of cycles omitted for each run can be found in table 2 .

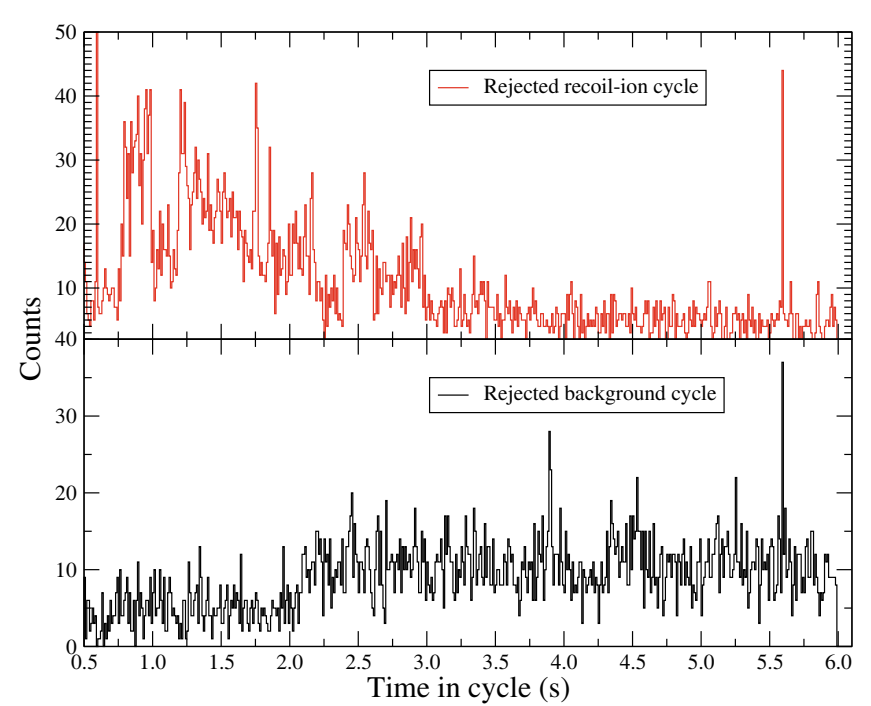

Fig. 7. (Colour online) Typical rejected recoil-ion cycle, where a spike occurs around $1.75 \mathrm{~s}$ in the middle of the $\mathrm{V}_{\text {ret. }} 2$ measurement (top) and rejected background cycle, where the background level changes after $2 \mathrm{~s}$ (bottom). The total number of cycles which were rejected are listed for each run in table 2 .

\subsubsection{Recoil-ion fits}

Independent amplitudes for all of the recoil-ion intensities, the beta intensity, and the implantation intensity were included in the fitting routine, as well as separate background parameters for each voltage applied to the retardation spectrometer during that run. Separate backgrounds were found to be necessary due to different amounts of background being focused on the MCP for different voltages applied to the retardation spectrometer, as can be seen from the variations in count rate between $t=3.0 \mathrm{~s}$ and $t=5.5 \mathrm{~s}$ in fig. 2. The components included when fitting particular portions of the cycle are listed and described in table 3 .

A typical experimental run, including both decay radiation and dedicated background cycles, is presented in fig. 8 with the resulting fit overlaid. Rather than fix the ion, beta, and implantation half-lives to those determined from fitting the dedicated half-life runs in sect. 3.1, those runs were fit simultaneously with each recoil-ion run listed in table 2 with single parameters for each of the ion, beta, and implantation half-lives common to all runs. This approach has the advantage that the uncertainties associated with the fitted half-life parameters are easily propagated to the uncertainties on the extracted intensities, which ultimately are the relevant parameters to be extracted.

A so-called "chop plot" is included in fig. 9, where it becomes clear that there are issues at the beginning of the experimental cycle. Though the exact cause for the slope during the first $50 \mathrm{~V}$ normalization measurement (see line two of table 3) is not fully understood, this feature is discussed in sect. 4.2 , and the first $50 \mathrm{~V}$ measurement for all runs has been omitted in the final analysis.

Following the fit, the recoil-ion intensities for the two retardation voltage values from each run are extracted 
Table 3. Components of the recoil-ion fit function. The first and last $10 \mathrm{~ms}$ of each region defined by the applied retardation potential are omitted from the fit to account for the finite charging time of the retardation spectrometer. Due to focusing effects each retardation voltage has its own background activity parameter, $B$, which is constrained by a dedicated background measurement cycle immediately following each recoil-ion cycle. The half-life parameters $\lambda_{\text {imp }}, \lambda_{\text {ion }}$, and $\lambda_{\beta}$ are common to the dedicated half-life runs described in sect. 3.1, and are determined by fitting those runs simultaneously with each recoil-ion run.

\begin{tabular}{|c|c|c|c|}
\hline $\begin{array}{l}\text { Time in } \\
\text { cycle }(\mathrm{s})\end{array}$ & $\begin{array}{l}\text { Retardation } \\
\text { potential }(\mathrm{V})\end{array}$ & Fit components & Description \\
\hline $0.76-0.79$ & 600 & $A_{\mathrm{imp}} \cdot e^{-\lambda_{\mathrm{imp}} t}+B_{600 \mathrm{~V}}$ & $\begin{array}{l}\text { Implantation activity only, before ions } \\
\text { are transfered to the decay trap. }\end{array}$ \\
\hline $0.81-0.99$ & 50 & $A_{50 \mathrm{~V}} \cdot e^{-\lambda_{\mathrm{ion}} t}+A_{\beta} \cdot e^{-\lambda_{\beta} t}+A_{\mathrm{imp}} \cdot e^{-\lambda_{\mathrm{imp}} t}+B_{50 \mathrm{~V}}$ & Ion normalization measurement at $50 \mathrm{~V}$. \\
\hline $1.01-1.19$ & 600 & $A_{\beta} \cdot e^{-\lambda_{\beta} t}+A_{\mathrm{imp}} \cdot e^{-\lambda_{\mathrm{imp}} t}+B_{600 \mathrm{~V}}$ & $\begin{array}{l}\text { Beta normalization measurement at } \\
600 \mathrm{~V} \text {, all ions are blocked. }\end{array}$ \\
\hline $1.21-1.59$ & $\mathrm{~V}_{\text {ret }} 1$ & $A_{V_{\mathrm{ret}} 1} \cdot e^{-\lambda_{\mathrm{ion}} t}+A_{\beta} \cdot e^{-\lambda_{\beta} t}+A_{\mathrm{imp}} \cdot e^{-\lambda_{\mathrm{imp}} t}+B_{V_{\mathrm{ret}} 1}$ & $\begin{array}{l}\text { First retardation voltage measurement, } \\
\text { actual value varies run to run. }\end{array}$ \\
\hline $1.61-1.99$ & $\mathrm{~V}_{\text {ret }} 2$ & $A_{V_{\mathrm{ret}} 2} \cdot e^{-\lambda_{\mathrm{ion}} t}+A_{\beta} \cdot e^{-\lambda_{\beta} t}+A_{\mathrm{imp}} \cdot e^{-\lambda_{\mathrm{imp}} t}+B_{V_{\mathrm{ret}} 2}$ & $\begin{array}{l}\text { Second retardation voltage measurement, } \\
\text { actual value varies run to run. }\end{array}$ \\
\hline $2.01-2.19$ & 50 & $A_{50 \mathrm{~V}} \cdot e^{-\lambda_{\mathrm{ion}} t}+A_{\beta} \cdot e^{-\lambda_{\beta} t}+A_{\mathrm{imp}} \cdot e^{-\lambda_{\mathrm{imp}} t}+B_{50 \mathrm{~V}}$ & Repeat ion normalization measurement. \\
\hline $2.21-2.39$ & 600 & $A_{\beta} \cdot e^{-\lambda_{\beta} t}+A_{\mathrm{imp}} \cdot e^{-\lambda_{\mathrm{imp}} t}+B_{600 \mathrm{~V}}$ & Repeat beta normalization measurement. \\
\hline $2.41-2.59$ & $\mathrm{~V}_{\text {ret }} 1$ & $A_{V_{\mathrm{ret}} 1} \cdot e^{-\lambda_{\mathrm{ion}} t}+A_{\beta} \cdot e^{-\lambda_{\beta} t}+A_{\mathrm{imp}} \cdot e^{-\lambda_{\mathrm{imp}} t}+B_{V_{\mathrm{ret}} 1}$ & $\begin{array}{l}\text { Repeat first retardation voltage } \\
\text { measurement. }\end{array}$ \\
\hline $2.61-2.79$ & $\mathrm{~V}_{\text {ret }} 2$ & $A_{V_{\mathrm{ret}} 2} \cdot e^{-\lambda_{\mathrm{ion}} t}+A_{\beta} \cdot e^{-\lambda_{\beta} t}+A_{\mathrm{imp}} \cdot e^{-\lambda_{\mathrm{imp}} t}+B_{V_{\mathrm{ret}} 2}$ & $\begin{array}{l}\text { Repeat second retardation voltage } \\
\text { measurement. }\end{array}$ \\
\hline $2.81-2.99$ & 50 & $A_{50 \mathrm{~V}} \cdot e^{-\lambda_{\mathrm{ion}} t}+A_{\beta} \cdot e^{-\lambda_{\beta} t}+A_{\mathrm{imp}} \cdot e^{-\lambda_{\mathrm{imp}} t}+B_{50 \mathrm{~V}}$ & Final ion normalization measurement. \\
\hline $3.01-3.19$ & 600 & $A_{\beta} \cdot e^{-\lambda_{\beta} t}+A_{\mathrm{imp}} \cdot e^{-\lambda_{\mathrm{imp}} t}+B_{600 \mathrm{~V}}$ & Final beta normalization measurement. \\
\hline
\end{tabular}

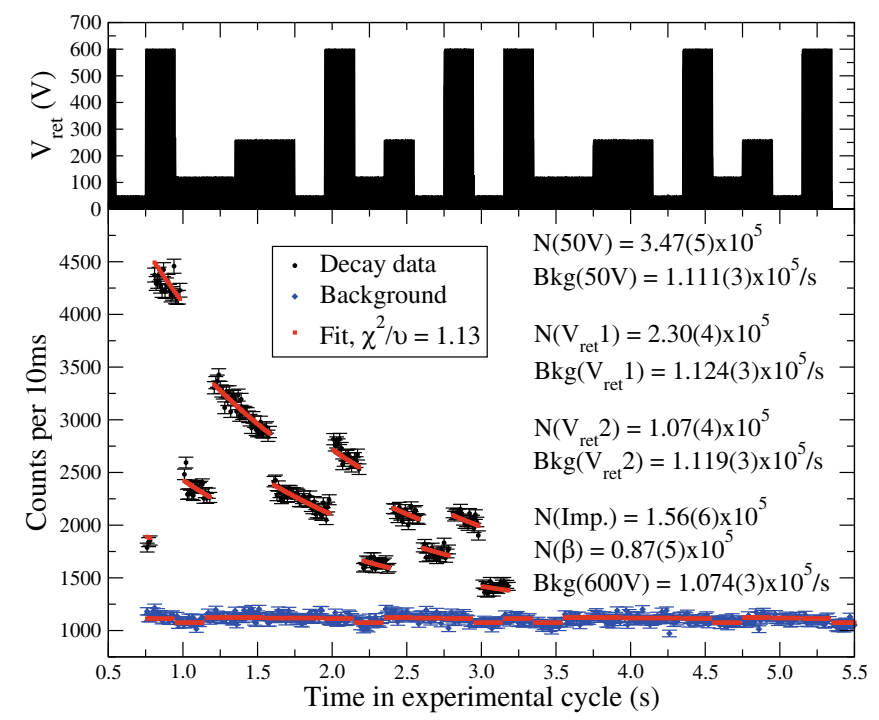

Fig. 8. (Colour online) Summed decay (black circles) and background (blue diamonds) cycles from run 96 with fit (red squares) overlaid. The top panel shows the retardation voltage sequence applied during this run.

and compared with a simulation (fig. 10). These intensities are expressed relative to the intensity measured at $50 \mathrm{~V}$ for each run, which allows for a normalization runto-run and removes any dependence on the initial number

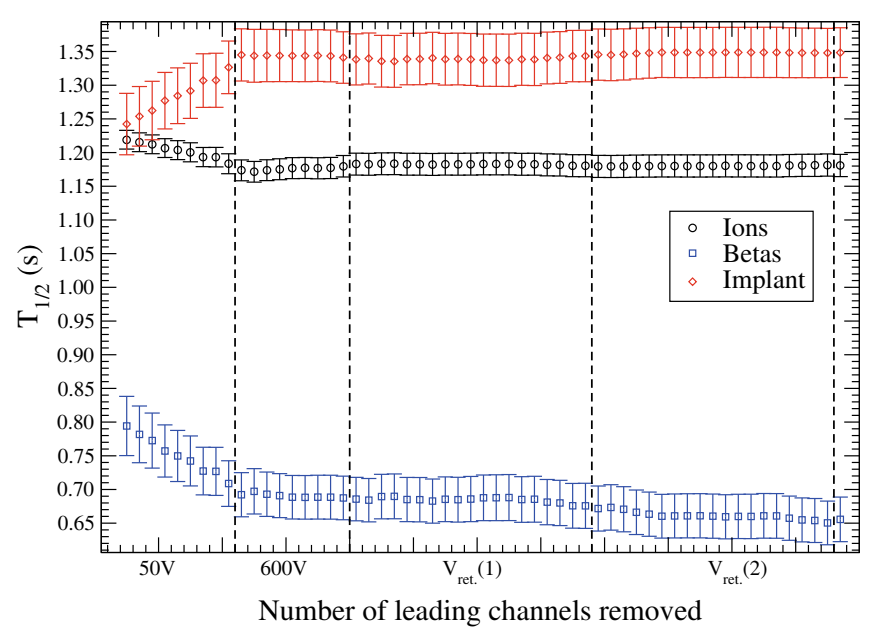

Fig. 9. (Colour online) Resulting half-lives for the ion, implant, and beta components for run 96 as a function of the number of leading channels removed. The vertical dashed lines indicate a change in the retardation potential.

of ions present in the traps. The simulations for the expected number of recoil ions at each experimental retardation voltage are performed using the SIMBUCA [30] and SimWITCH $[51,52]$ simulation packages. The SIMBUCA simulation package models the behaviour of multiple ions in a Penning trap, and is used to obtain the initial position and velocity of the ${ }^{35} \mathrm{Ar}$ ions stored in the decay trap 


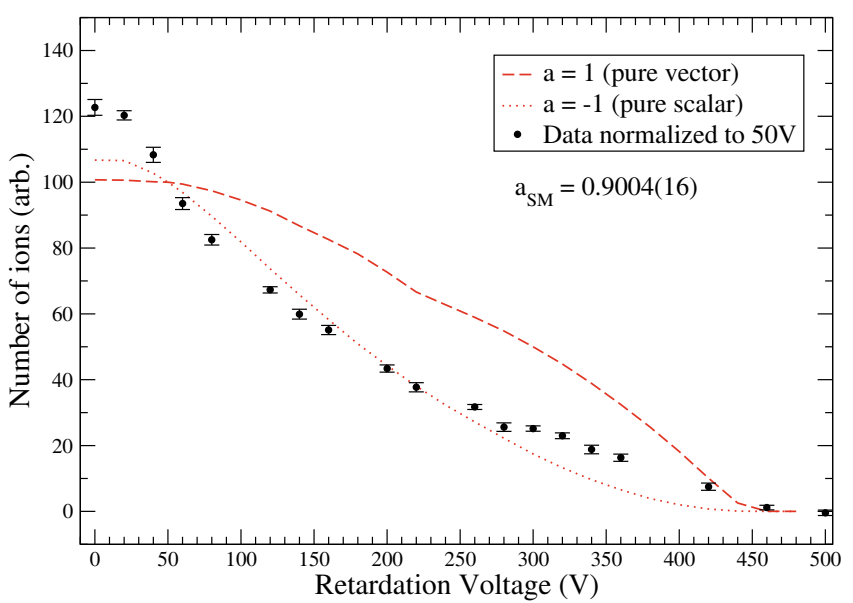

Fig. 10. (Colour online) Experimental recoil-ion intensities generated in the analysis described in sect. 3.2.2 and normalized to $50 \mathrm{~V}$ (black circles), and simulated values assuming pure vector (red dashed line) and pure scalar (red dotted line) interactions. The standard model value for the $\beta-\nu$ correlation parameter in ${ }^{35} \mathrm{Ar}$ decay is 0.9004(16) [32], and the experimental data would thus be expected to closely follow the $a=1$ curve. The error bars for the experimental data reflect statistical uncertainties only.

prior to their decay. Following decay, the recoiling daughter nucleus' trajectory is tracked through the retardation spectrometer, re-acceleration section, and ultimately to the surface of the MCP detector using the SimWITCH tracking program.

As is evident from fig. 10, the recoil-ion data is completely at odds with the Standard-Model value for the $\beta-\nu$ correlation in ${ }^{35} \mathrm{Ar}$ decay. Not only do some data points fall below the possible lower limit imposed by a pure scalar interaction, but the excess of counts at low voltage also indicates that further systematics need to be treated in order to reconcile this data set with the simulations. A possible source for this discrepancy is given in sect. 4.2.

\subsubsection{Independent cycle analysis}

An independent analysis with respect to the one detailed in sect. 3.2.2 was also carried out using ROOT and the Minuit fitting routine [43]. Apart from the use of a separate fitting routine, several other features of the experimental spectra were incorporated into the fit.

\section{i) Magnetron motion}

In fig. 11 a portion of the experimental cycle slightly before and immediately after the transfer of the ions from the cooler trap to the decay trap at $0.8 \mathrm{~s}$ is displayed with a fine binning of $0.1 \mathrm{~ms}$. With this resolution it is clear that the signal from the decay trap is modulated with an oscillation of $172 \mathrm{~Hz}$ (period of $5.8 \mathrm{~ms}$ ), which is approximately equal to the magnetron frequency of argon in a $6 \mathrm{~T}, 15 \mathrm{~V}$ Penning trap of $180 \mathrm{~Hz}$. Since this oscillation does not react to changes in the retardation voltage, i.e.

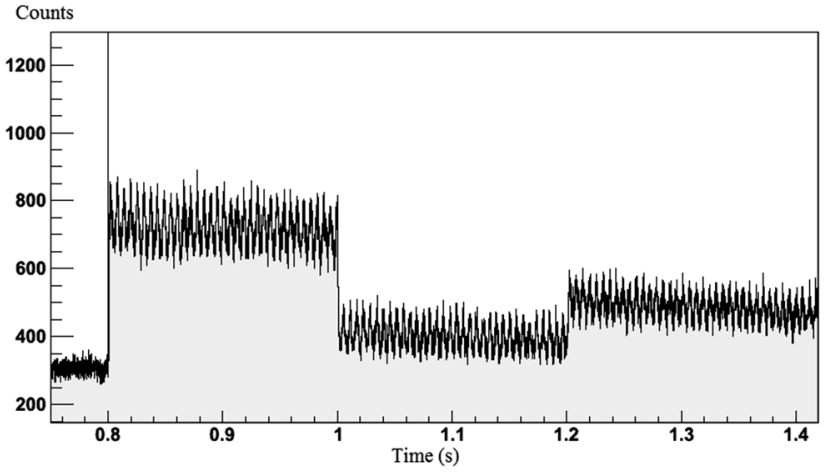

Fig. 11. Portion of the experimental cycle immediately following the transfer of the ions from the cooler trap to the decay trap. A bin size of $0.1 \mathrm{~ms}$ is used to resolve the magnetron oscillation, which has a period of approximately $5 \mathrm{~ms}$. Note that the magnetron oscillation is absent from the signal below $t=0.8 \mathrm{~s}$ (i.e. the time of the transfer from the CT trap to the DT trap shown in fig. 2), and therefore must be associated with activity from the trap, while the amplitude of the oscillation does not change when the retardation voltage is switched at $1 \mathrm{~s}$ and $1.2 \mathrm{~s}$.

the amplitude does not change at $1 \mathrm{~s}$ in the cycle when the retardation voltage is switched from $50 \mathrm{~V}$ to $600 \mathrm{~V}$, and persists in regions which are free of recoil ions, it must be associated with the $\beta$ particles coming from the decay trap. The fact that an oscillation with the magnetron frequency is visible in the activity suggests that the ion cloud is not perfectly centered in the decay trap. It is possible that there is a small misalignment between the electric and magnetic fields, an effect which was confirmed in a previous experimental campaign [51], and although steps were taken to correct the misalignment, it appears that it has not been completely eliminated.

The effect of the magnetron oscillation was modeled in the fit function by an underdamped harmonic sinusoidal oscillator with frequency $f$ and phase $\phi$ multiplied by a decreasing exponential with half-life $T_{\text {mag. }}$ and amplitude $A_{\text {mag. }}$ The half-life that was fit to the decay component of the oscillation lies around $0.6 \mathrm{~s}-0.7 \mathrm{~s}$ for most runs, in excellent agreement with the $\beta$ half-life measured in the dedicated half-life runs, and further supports the conclusion that the magnetron oscillation is solely associated with the $\beta$ activity.

\section{ii) Desorption}

Between $3.20 \mathrm{~s}$ and $3.21 \mathrm{~s}$ in the measurement cycle the ions are ejected from the center of the decay trap with a dipole excitation of $180 \mathrm{~Hz}$ and an amplitude of $10 \mathrm{~V}$, pushing them to larger radii and onto the electrode walls of the trap, while the trapping potential is simultaneously switched to enable upstream ejection toward the pumping diaphragm. The remaining low-energy ${ }^{35} \mathrm{Ar}$ ions undergo charge exchange with these surfaces, neutralize, and then desorb back into the trap volume. The growth in activity after $3.21 \mathrm{~s}$ as seen in fig. 12 is most likely the $\beta^{+}$ particles emitted from the neutralized ${ }^{35} \mathrm{Ar}$, rather than 


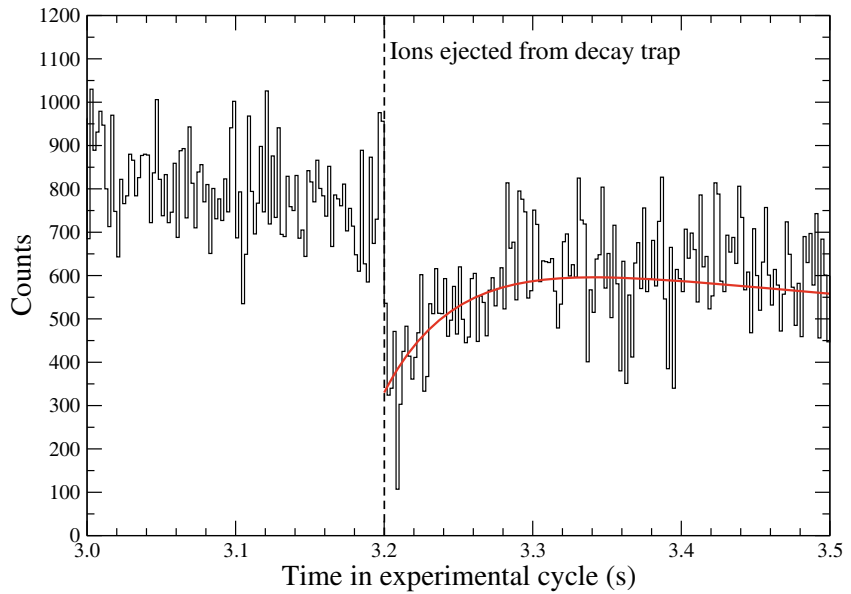

Fig. 12. Portion of the experimental cycle immediately before and after the ejection of the ions from the decay trap via a radial excitation. In order to illustrate the effect all the experimental runs have been summed with a bin size of $2 \mathrm{~ms}$, and a desorption curve generated from eq. (2) with $\lambda_{d}=1 \mathrm{~s}^{-1}$ and $\lambda_{p}=20 \mathrm{~s}^{-1}$ is overlaid.

${ }^{35} \mathrm{Ar}$ recoils, as the spectrometer is switched from $50 \mathrm{~V}$ to $600 \mathrm{~V}$ at $3.4 \mathrm{~s}$, yet there is no visible change in the activity at this point. Assuming that the ${ }^{35} \mathrm{Ar}$ that interacts with the walls can either decay in the walls or desorb back into the trap volume, and the neutralized ${ }^{35}$ Ar which desorbs can either decay or be pumped away, the differential equation for the desorbed ${ }^{35} \mathrm{Ar}$ is

$$
\frac{\mathrm{d} N_{d}}{\mathrm{~d} t}=\lambda_{d} N_{0} e^{-t\left(\lambda+\lambda_{d}\right)}-\lambda N_{d}-\lambda_{p} N_{d},
$$

with $N_{0}$ the initial amount of adsorbed ${ }^{35} \mathrm{Ar}, \lambda_{d}$ the desorption rate, $\lambda_{p}$ the pumping rate, and $\lambda$ the nuclear decay constant of ${ }^{35} \mathrm{Ar}$. Incorporating this model into the fit function nicely reproduces the data around $3.2 \mathrm{~s}$, and results in values for the rates of $1-4 \mathrm{~s}^{-1}$ for $\lambda_{d}$ and $6-20 \mathrm{~s}^{-1}$ for $\lambda_{p}$.

\section{iii) Free implantation half-life}

By incorporating the desorption of the ions into the fitting function it is possible to extend the analysis of the experimental spectrum into the region between $3.2 \mathrm{~s}$ and $5.6 \mathrm{~s}$ (see fig. 2). In this portion of the cycle the activity is dominated by the ions implanted in the MCP during the loading of the traps, and in this analysis the half-life for this activity was thus left free to be determined by the fit. The weighted average of the implantation half-lives measured in these runs was found to be $1.282(11) \mathrm{s}$, in excellent agreement with the value of $1.311(31) \mathrm{s}$ obtained from the dedicated half-life runs (see table 1 ), and provides a complementary determination of this important experimental parameter.

While the implantation half-life deduced from the recoil runs themselves is nearly a factor of three more precise than that which is determined from the dedicated half-life runs, the need to incorporate the desorption model, and the explicit treatment of the magnetron oscillation rather

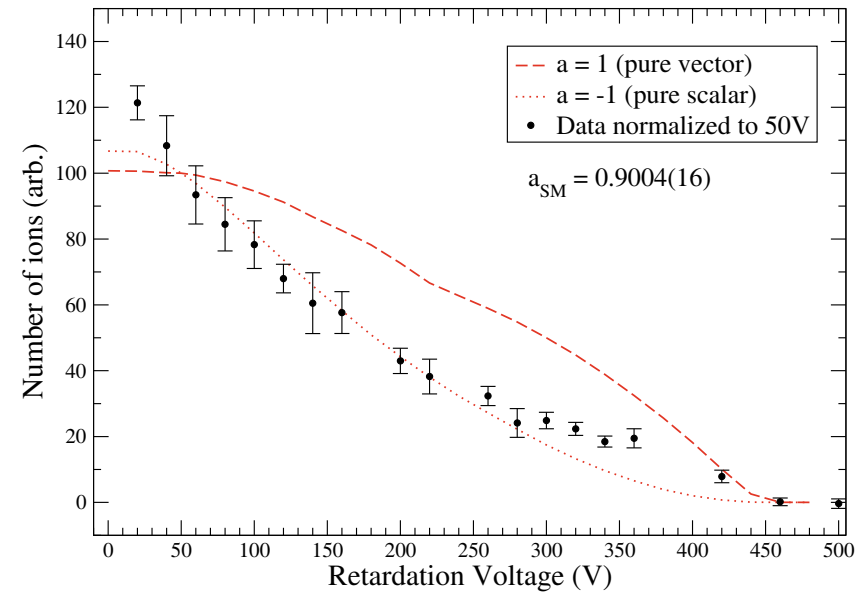

Fig. 13. (Colour online) Experimental recoil-ion intensities normalized to $50 \mathrm{~V}$ (black circles), and simulated values assuming pure vector (red dashed line) and pure scalar (red dotted line) interactions. The standard model value for the $\beta-\nu$ correlation parameter in ${ }^{35} \mathrm{Ar}$ decay is $0.9004(16)$ [32]. The error bars for the experimental data reflect statistical uncertainties only. These data result from the analysis described in sect. 3.2.3, and with the exception of the inflated error bars are very similar to the data presented in fig. 10, despite the incorporation of additional features into the fitting routine.

than re-binning to average it out, result in somewhat less precise values for the recoil-ion amplitudes as displayed in fig. 13. The agreement between the two independent methods for the recoil-ion amplitudes themselves, however, is quite good and suggests that the deficiency in the curve for the recoil-ion amplitudes lies in some as-yet-untreated systematic effect, rather than in any deficiency in either of the particular methods employed in the experimental analysis.

\section{Systematics}

In a recent publication presenting the first results for $a_{\beta \nu}$ in ${ }^{35} \mathrm{Ar}$ decay with WITCH [25], a table was included outlining the main sources of systematic uncertainty in the value for $a_{\beta \nu}$ determined with this setup, and how well these sources were under control at the time. As the current data set represents a vast improvement in overall statistics, number of retardation potentials sampled, and timing resolution, several new effects which could not be identified from the previous data, such as the magnetron oscillation and desorption etc., have been uncovered. Two additional effects, which have the largest impact on the extraction of $a_{\beta \nu}$, will be presented in this section.

\subsection{Energy-dependent MCP efficiency}

The recoil ions with enough kinetic energy to overcome the retardation potential are focused via a series of electrodes and an Einzel lens onto the large diameter MCP for detection (see sect. 2). Since the recoil ions typically have 


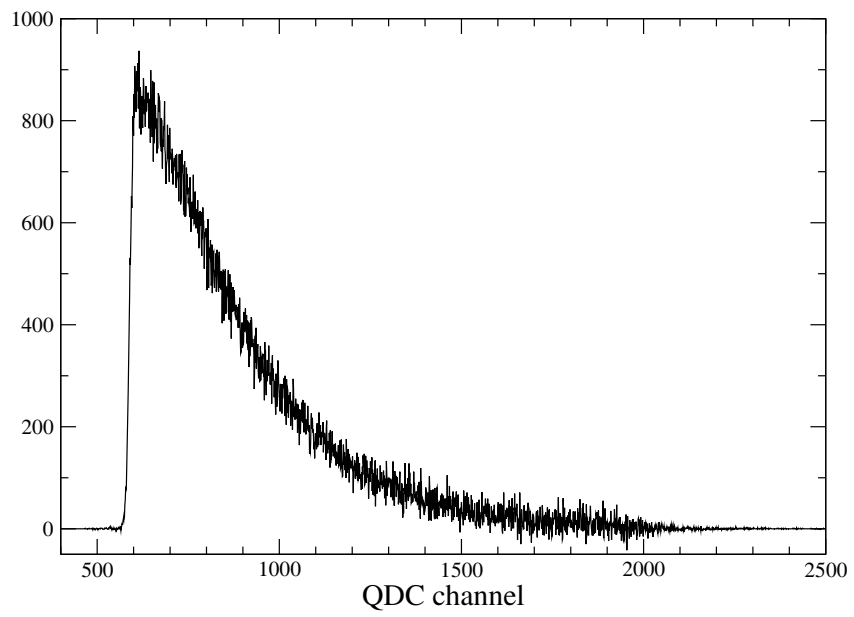

Fig. 14. Background-subtracted QDC distribution for events in the cycle between $0.8 \mathrm{~s}$ and $3.2 \mathrm{~s}$. The sharp edge is uncharacteristic of a normal MCP pulse height distribution for ions, which should have a Gaussian shape.

very low kinetic energies $\left(E_{\max } \approx 452 \mathrm{eV}[25]\right)$, they are re-accelerated to $3.2 \cdot q \mathrm{keV}$, where $q$ is the charge state of the ion, before impinging on the MCP surface. Typically, MCP detectors provide good efficiency for ions above $3 \mathrm{keV}$ [44], however, they can be affected by variations of the efficiency across the surface as well as gradual deterioration caused by impinging energetic particles, resulting in decreased efficiency. Careful investigation of the pulse height distributions for recoil events from this experiment (fig. 14) reveal a skewed Gaussian shape characteristic of a damaged MCP, which has been shown to correlate with reduced detection efficiency [44].

Due to the charge-dependence of the re-acceleration section, the lowest charge states of the recoil-ion distribution will be most affected by an energy-dependent efficiency for detection. Such an effect will alter the measured shape of the recoil-ion intensities vs. applied retardation potential because the different charge states (ranging from $1^{+}$to $5^{+}$[53]) will not be uniformly distributed throughout the full recoil-ion energy range when measured with a retardation spectrometer. For example, while a $1^{+}$recoil ion with $300 \mathrm{eV}$ will pass a $250 \mathrm{~V}$ potential and thus contribute to the measured count rate, a $2^{+}$ion with $300 \mathrm{eV}$ will not. Even though the $2^{+}$recoil ion has an excess of kinetic energy above the retardation potential, its charge state of $2^{+}$ensures it effectively experiences twice the applied potential, in this case $500 \mathrm{~V}$, and so would not contribute to the count rate for a retardation potential of $250 \mathrm{~V}$.

The upper half of the recoil-ion energy distribution will thus only contain $1^{+}$ions, and these ions will be reaccelerated towards the MCP with at most half the energy of the rest of the ions. If the MCP has an energydependent efficiency for detection, the upper portion of the recoil-ion intensity plot will thus be most affected. A reduced efficiency was modeled by fitting the experimental data using the simulated data assuming the StandardModel value for $a_{\beta \nu}$ in ${ }^{35} \mathrm{Ar}$ decay, and using coefficients

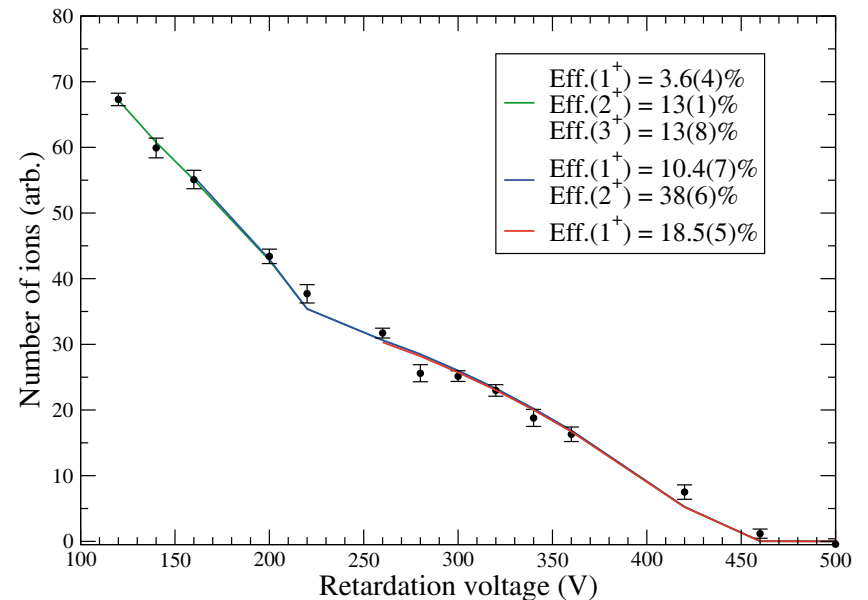

Fig. 15. (Colour online) Fit to the $1^{+}$(red), $1^{+}$and $2^{+}$(blue), and $1^{+}, 2^{+}$, and $3^{+}$(green) regions of the recoil-ion amplitudes $v s$. retardation voltage assuming the standard model value for $a_{\beta \nu}$ in ${ }^{35} \mathrm{Ar}$ decay and allowing for a variable detection efficiency for each of the charge states.

for the detection efficiency of each charge state as parameters. The results of these fits, which suggest this as a valid hypothesis only if the efficiency is reduced by more than approximately $80 \%$, are illustrated in fig. 15 . In order to verify and quantify the reduction, if any, in ion-detection efficiency as a function of energy for our MCP, dedicated offline measurements were performed following the beam time.

\subsubsection{Experimental setup}

The MCP efficiency measurements were performed at LPC Caen, where existing infrastructure for testing MCPs was installed [44]. The setup consisted of a cross-shaped vacuum chamber with the WITCH MCP and an ion source (IS) opposite one another. The chamber was evacuated by a turbomolecular pump connected to a prevacuum scroll pump, with typical pressures of $10^{-7}$ mbar achieved. The ion source stack (fig. 16) consisted of an exchangeable heat-activated pellet that produces positive alkali ions for beam manipulation, in this case ${ }^{6} \mathrm{Li},{ }^{23} \mathrm{Na}$, and ${ }^{85} \mathrm{Rb}$. The IS was biased to a high positive potential $(1.7-2.7 \mathrm{kV})$ to accelerate the ions, with one lens electrode for focusing and three steering electrodes available for moving the beam spot across the MCP surface. The ion source stack was also fitted with an attenuating grid (factor $10^{4}$ ) to reduce the beam intensity to a few $\mathrm{kHz}$ of event frequency registered by the MCP in order to avoid saturation and damage. The grid was also used to measure the beam current via a Keithley Systems Electrometer, and was enclosed in a pseudo Faraday cup that captured most of the secondary electrons. This device deviates from a true Faraday cup in that a hole is present at the end in order to allow the ions to pass through to the MCP. This resulted in a loss of some of the secondary electrons produced in the grid and causes a systematic error in the current nor- 


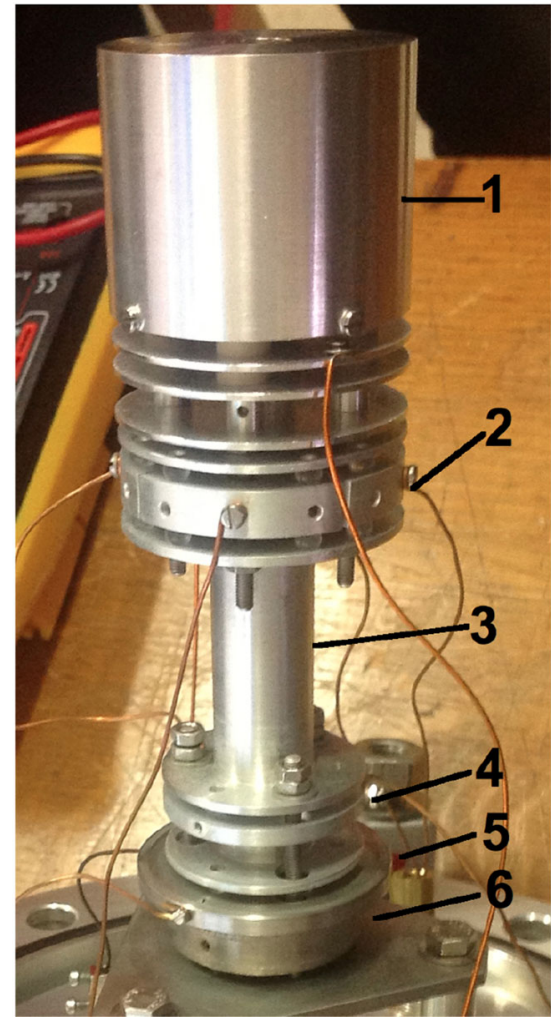

Fig. 16. (Colour online) The ion source stack. Faraday cup with an opening on top (1), beam steering electrodes (2), drift electrode (3), electrostatic lens (4), extraction electrode (5) and ion source heater (6) are indicated.

malizations. For this reason only relative efficiencies are reported here.

Since the secondary emission properties depend on the ion-source energy, the ion source was maintained at a constant potential $(2.7 \mathrm{kV}$ or $1.7 \mathrm{kV})$ to minimize the variation of secondary electron emission intensity, resulting in a more reliable run to run normalization. The MCP's voltage divider was thus modified to allow independent settings of the front and back plate voltages, while keeping the voltage over the plates constant at $3150 \mathrm{~V}$ as was the case during the online experiment. This provided incident ions in an energy range of $1.7 \mathrm{keV}$ to $6.7 \mathrm{keV}$, which encompasses the re-accelerated energies of the $1^{+}$and $2^{+}$ ${ }^{35} \mathrm{Cl}$ ions from the online experiment.

\subsubsection{Measurements}

Each measurement for a particular ion species at a particular kinetic energy was done with a beam spot of approximately $1 \mathrm{~cm}$ radius focused on the region of the MCP onto which the recoil ions were focused during the ${ }^{35} \mathrm{Ar}$ beam time. In order to account for background, particularly from neutralized ions passing through the attenuation grid, the beam spot was steered to the edge of the $\mathrm{MCP}$ following each ion measurement and a background
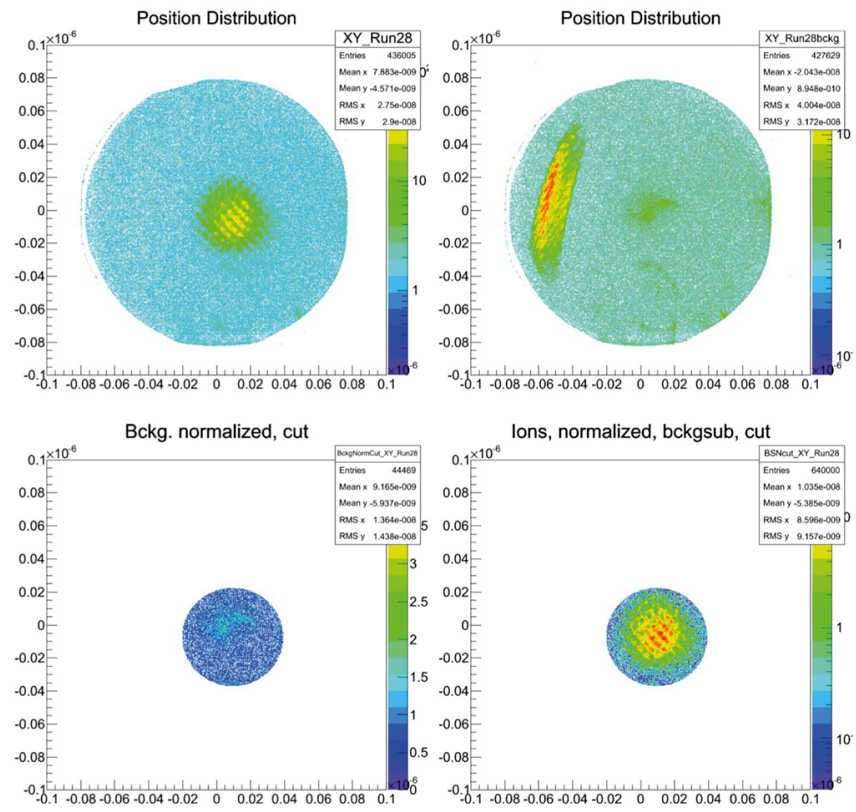

Fig. 17. (Colour online) Overview of the background subtraction process. Top-left: ion measurement at the approximate position of the beam spot during the online ${ }^{35} \mathrm{Ar}$ experiment. Top-right: measurement of the background contribution at the position of the beam spot. The ions are deflected to the MCP rim. Bottom-left: a cut on the position of the beam spot in the background measurement showing a small concentration of neutral atoms which are not affected by the electrostatic deflectors. Bottom-right: the final histogram of the normalized beam spot with the neutral background subtracted.

measurement was taken. This series of efficiency measurement followed by background measurement was repeated for each acceleration voltage to verify reproducibility.

In addition to the ion measurements at varying incident energy, calibration measurements were also performed to ensure linearity of the grid current vs. recorded ion intensity on the MCP. While the slope of the collected charge vs. number of ions is generally linear, there is much more scatter in the data points when the front plate of the MCP is fixed to $0 \mathrm{~V}$, and for the same amount of charge collected by the grid many more counts are recorded on the MCP. This is likely caused by an excess of electrons coming from the grid, which are normally repelled when the MCP front plate is set to a negative potential, but not when set to $0 \mathrm{~V}$. This effect precludes the possibility of an absolute normalization, but should not affect the background subtraction.

The 2D histograms for the beam spot and background measurements were constructed and normalized to the total charge collected on the grid. A cut was made on the beam spot position and the respective cut on the background was subtracted, as the background contains ions as well as neutral particles originating from the ion source that are not affected by the electrostatic steering elements (see fig. 17). These normalized and background-subtracted count rates for each ion species were then compared as a function of accelerating potential. 


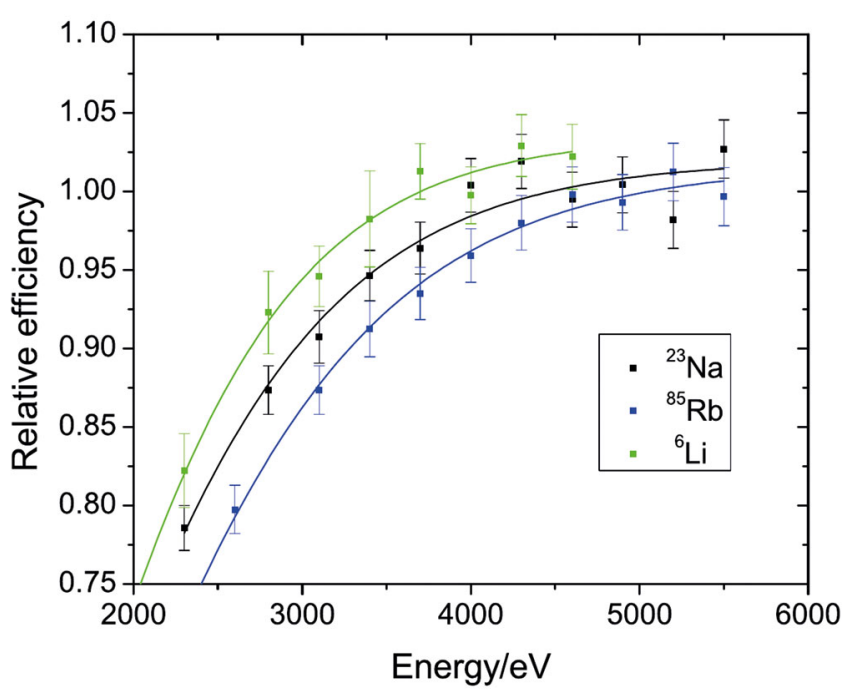

Fig. 18. (Colour online) Relative detection efficiency for the WITCH MCP as a function of incident ion energy. The mass scaling of the different curves conforms well with theory [54], and indicates that the WITCH MCP has a detection efficiency for ${ }^{35} \mathrm{Cl}^{1+}$ which is no less than $85 \%$ for ${ }^{35} \mathrm{Cl}^{2+}$ recoil ions.

\subsubsection{Results}

For all three ion species the count rate was found to increase up to an ion energy of approximately $4.2 \mathrm{keV}$ before saturation, a significantly higher value than $3 \mathrm{keV}$ found in ref. [44]. The count rates for ${ }^{23} \mathrm{Na}$ and ${ }^{85} \mathrm{Rb}$ were found to be stable in the plateau region up to $5.5 \mathrm{keV}$, while the data in the region from $5.5 \mathrm{keV}$ to $6.7 \mathrm{keV}$ fluctuated significantly about the saturation plateau. For these data the points from $4.25 \mathrm{keV}$ to $5.5 \mathrm{keV}$ were thus used to estimate the saturation efficiency in the plateau region. The data for ${ }^{6} \mathrm{Li}$ was found to be much more erratic in the plateau region, likely due to instabilities in the ion source, and thus only the point at the start of the plateau at $4.5 \mathrm{keV}$ was used for normalization in this case. The resulting relative efficiency curves for each ion species is displayed in fig. 18 .

From the relative efficiency curves it can be seen that a constant efficiency as a function of incident energy is not achieved before $4.5 \mathrm{keV}$, and exhibits a definite slope in the $3.1 \mathrm{keV}$ to $3.6 \mathrm{keV}$ energy region corresponding to the energy range for ${ }^{35} \mathrm{Cl}^{1+}$ recoils. From these data it is difficult to extract a precise value for the reduction in detection efficiency over this range, but it is clearly no worse than $85 \%$ of the efficiency for the $2^{+}$and higher charge states which would be incident with a minimum energy of $6.2 \mathrm{keV}$. Although the normalization procedure was found to be unsuitable for a precise determination of the absolute efficiency of the MCP, the fact that all count rates plateaued after approximately $4.5 \mathrm{keV}$ suggests that beyond this energy ions are detected by the MCP with full efficiency.

The effect of a reduced efficiency for the detection of $1^{+}$ ions is incorporated into the simulated data in fig. 19, as it is impossible to separate charge states in the experimental spectra. It is evident that adopting a relative efficiency of

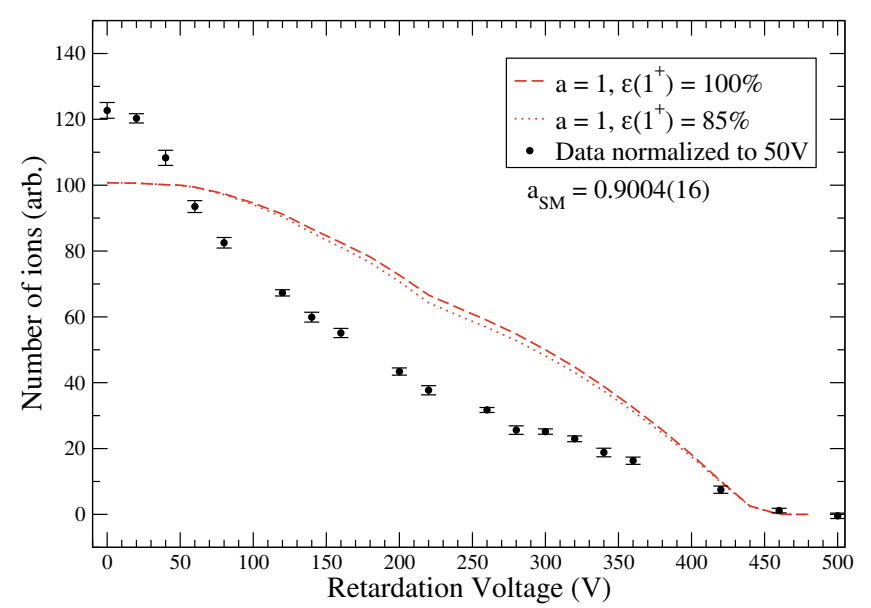

Fig. 19. (Colour online) Experimental recoil-ion intensities normalized to $50 \mathrm{~V}$ (black circles) and simulated curves for $a_{\beta \nu}=1$ assuming a 100\% (red dashed line) and 85\% (red dotted line) relative detection efficiency for the ${ }^{35} \mathrm{Cl}^{1+}$ recoil ions.

$85 \%$ for the $1^{+}$recoil ions is not sufficient to resolve the observed discrepancy between the experimental data and the SM value for $a_{\beta \nu}$ in ${ }^{35} \mathrm{Ar}$ decay.

\subsection{Radiation-induced background}

It has been observed in other experiments utilizing retardation spectrometers that neutral rest gas, or buffer gas in the case of a Penning trap, can become ionized when radiation is introduced into the system $[55,56]$. Such a background will be absent from any dedicated background measurements, as its creation requires the presence of radiation to ionize it.

Identifying such a radiation-induced background component, which may or may not be time-dependent, with offline measurements is extremely challenging as it would not only require several radioactive sources of varying intensity which can closely mimic the characteristics of the online activity, but the exact same vacuum and buffer gas conditions encountered in the online experiment must be reproduced as well. Moreover, it is impossible with an offline radioactive source to mimic the spatial distribution of ions in the trapped ion cloud. Such an intensive systematic investigation has not been possible with the current WITCH setup, thus Monte Carlo simulations were performed in order to investigate the plausibility of such a background producing the type of systematic effect which is observed in the present data set.

\subsubsection{Monte Carlo for recoil-ion runs}

Recoil-ion runs were simulated with a $\mathrm{C}++$ program that produced the time between subsequent decay events according to a random distribution $-\ln (r) / \lambda$, where $r$ is a random 48 bit floating-point number between 0 and 1 generated by the drand48() routine. The experimental halflives $T_{\text {imp }}, T_{\text {beta }}$, and $T_{\text {ion }}$ from sect. 3.1 were used, while 


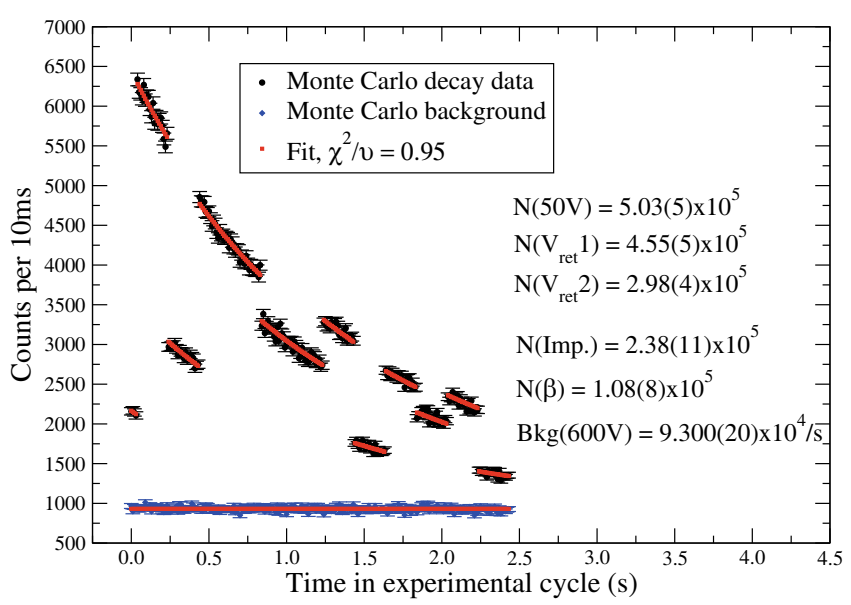

Fig. 20. (Colour online) A Monte Carlo generated version of the recoil-ion run presented in fig. 8, assuming all of the components present in the fit function of table 3 , with the recoil ions in proportion according to the SM value of $a_{\beta \nu}$ for ${ }^{35}$ Ar decay.

the beta and implantation activities were chosen to be in the same proportion relative to the recoil-ion intensities as determined from fitting the experimental data.

The recoil-ion amplitudes for the range of retardation voltages probed in the experiment were determined using SimWITCH assuming the SM value for $a_{\beta \nu}$ in ${ }^{35} \mathrm{Ar}$ decay. In this way SM recoil-ion runs were created assuming only the presence of components already defined in the fitting model from table 3 . Fitting such a spectrum with the function defined in table 3 will thus return the SM value for $a_{\beta \nu}$ (fig. 20).

Before proceeding to investigate the influence of unaccounted for background on the extracted values of the recoil-ion amplitudes, the generated decay curves were fit using the function in table 3 to ensure the input values for the half-lives and amplitudes were returned. Both the ROOT-based and C-based fitting routines described in sect. 3.2 were found to reliably extract the simulated values to within $1 \sigma$ for all simulated runs.

\subsubsection{Background models}

In order to test the hypothesis that the systematic deviation from the SM seen in the recoil-ion amplitude versus retardation potential plot of fig. 10 is caused by an unaccounted for background component which is present only when ions are in the system, two separate background models where considered:

- a background constant in time throughout the ion cycle, but absent in the background cycle, with an energy spread according to a Gaussian distribution with mean and $\sigma$ in the $0 \mathrm{eV}$ to $200 \mathrm{eV}$ range;

- a background time-correlated to the decay of the ion cloud, and hence absent in the background cycle, with an energy spread according to a Gaussian distribution with mean and $\sigma$ in the $0 \mathrm{eV}$ to $200 \mathrm{eV}$ range.

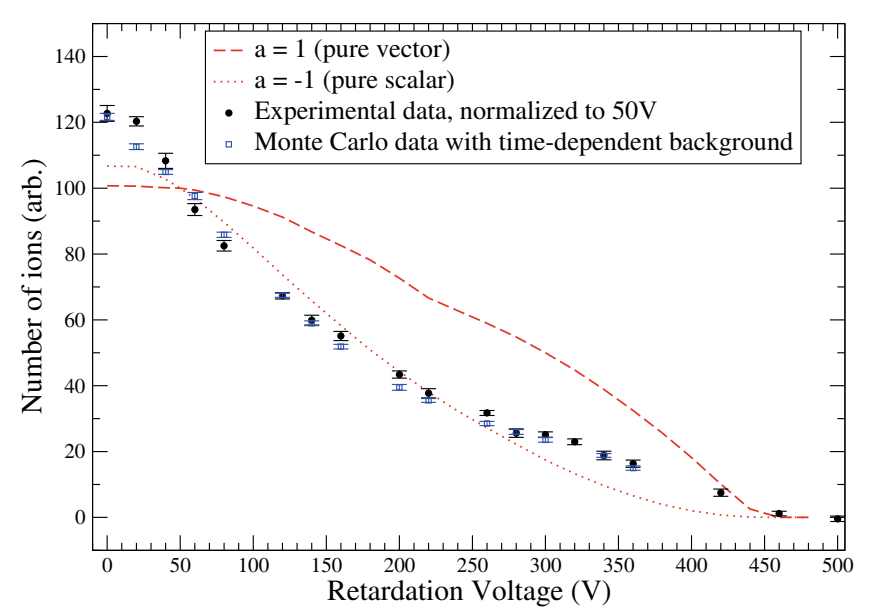

Fig. 21. (Colour online) Experimental recoil-ion amplitudes from fig. 10 (black circles) and the recoil-ion amplitudes extracted from Monte Carlo data (blue open squares) assuming the SM value for $a_{\beta \nu}$ in ${ }^{35} \mathrm{Ar}$ decay and the presence of a timedependent background, which is unaccounted for in the fitting routine.

The choice to confine the distributions to this particular energy range stems from the fact that the activity in the spectrometer is seen to go to zero at the end-point of the ${ }^{35} \mathrm{Ar}$ recoil energy, and thus all background must be below this energy as well. Energy distributions were created with mean and $\sigma$ spanning the range described above, and for each combination the energy distribution was integrated to include all ions with energies higher than the particular retardation voltage being simulated. This approach assumes that the background ions are created somewhere between the decay trap and the analysis plane, so that only those ions with enough energy to overcome the retardation barrier are counted in the cycle. This radiation-induced background component was then added to the simulated SM decay curves, which were then fit again with the function from table 3 to see how the resulting recoil-ion amplitudes are affected by this additional source of background which is not explicitly included in the fit function.

Fits of the generated spectra revealed that it is possible to find a single energy distribution of background ions that reproduces the experimentally observed spectrum fairly well (see fig. 21). The best agreement was found for a time-dependent energy distribution with mean of $50 \mathrm{eV}$ and $\sigma$ of $100 \mathrm{eV}$. Reasonable agreement was also achieved with a constant energy distribution, but the resulting reduced chi-squares are much too high $(\sim 20)$, while the reduced chi-squares for the time-dependent distribution were on the order of 1.1-1.2, in excellent agreement with the range of reduced chi-squares from the fits to the experimental data, and further supporting the hypothesis of an unaccounted for time-dependent background component in the decay data.

The fact that the addition of a reasonable Gaussiandistributed time-dependent background to the Monte Carlo data not only reproduces the experimental amplitudes, but the $\chi^{2} / \nu$ from the fits as well, strongly supports 
the hypothesis that there is a time-dependent background component in the data set which is currently not accounted for in our fitting model. Unfortunately the procedure used to identify and categorize this background relies on the assumption of the SM value for $a_{\beta \nu}$ in ${ }^{35} \mathrm{Ar}$ decay, negating the possibility of using this information to extract the value of $a_{\beta \nu}$ from the current data set. Detailed systematic studies of this effect in the WITCH spectrometer would need to be performed to further characterize this background before another data set could be taken with the hope of yielding an accurate value for $a_{\beta \nu}$ in ${ }^{35} \mathrm{Ar}$ decay. Improving the conditions which lead to such a background, however, is of even more importance. In particular the vacuum conditions in the system, which were two orders of magnitude worse in this measurement than during the previous campaign [25] where this effect was below the level of statistical uncertainty, would need to be drastically improved to minimize the impact of this systematic on the extracted value of $a_{\beta \nu}$.

\section{Summary and conclusions}

The first high-statistics high-resolution recoil-ion data for ${ }^{35} \mathrm{Ar}$ decay was taken in November 2012 at CERNISOLDE with the WITCH spectrometer. A number of electronics upgrades, most notably moving from an MCS and Lab View-based data acquisition system to the FASTER DAQ developed at LPC Caen, enabled the detection of decay products on an event-by-event basis with negligible dead time. Despite low production yields of ${ }^{35} \mathrm{Ar}$ and relatively poor transmission efficiency to the WITCH Penning traps, well over 25 million recoil events were registered over the course of the experiment, although poor background conditions resulted in an acceptable signal-tonoise ratio for only $13 \%$ of the data.

Fitting the highest-quality recoil-ion runs and extracting the recoil-ion amplitudes yields a result for $a_{\beta \nu}$ in ${ }^{35} \mathrm{Ar}$ decay which is completely at odds with the SM prediction, indicating further sources of systematic effects not previously considered with this apparatus. A worn and damaged MCP detector, which resulted in a reduced detection efficiency for $1^{+}$recoil ions, was found to be the second largest systematic effect affecting this data set. Precisely and accurately characterizing the absolute energydependent efficiency of this MCP in order to correct for the damage has proven to be very difficult, and in the future a careful assessment of the state of the MCP prior to the experiment, with replacement an option in case of damage, would be warranted.

The largest systematic effect observed in the present data set was found to be a time-dependent radiationinduced background. Due to the nature of such a background, which is known to affect retardation spectrometers, direct observation was not possible, and its presence was thus inferred via Monte Carlo simulations which verified that reasonable assumptions about the properties of such a background would indeed have precisely the effect on the extracted recoil-ion amplitudes as is observed in the present data set. As verification of the presence of such a background depended on the assumption of the SM value for $a_{\beta \nu}$ in ${ }^{35} \mathrm{Ar}$ decay, correcting for this background and extracting an experimental value for $a_{\beta \nu}$ in ${ }^{35} \mathrm{Ar}$ decay is not possible with the present data set. While this background could be studied and characterized in dedicated offline measurements, the impact of such a background depends critically on the conditions in the spectrometer, which were very poor during this beamtime compared to previous campaigns, and thus correcting for this background in the present data is not possible.

Other retardation spectrometers have had some success treating these backgrounds via careful offline study, and using the results of these studies to minimize their impact, but they have also found that completely eliminating a radiation-induced background component is not feasible [56]. With other experiments such as LPCTrap [17] making great strides in precision $a_{\beta \nu}$ measurements, the massive effort and time commitment that would be involved in characterizing and minimizing a radiation-induced background at WITCH, and the strong competition for radioactive beam time at ISOLDE which would be required in order to pursue such studies, the collaboration has decided that the WITCH spectrometer will be repurposed for other fundamental symmetries studies and will no longer be focused on recoil-ion measurements for the extraction of $a_{\beta \nu}$ for ${ }^{35} \mathrm{Ar}$ decay.

The authors would like to thank the ISOLDE Collaboration and the ISOLDE operators. This work was supported by FWO-Vlaanderen (Belgium), GOA/2010/10 (BOF-K.U. Leuven), IUAP-Belgian State Belgian Science Policy - (BriX network P6/23), German BMBF-Verbundforschung (Foederkennzeichen 06MS9151I), Grant LG13031 and LG15052 of the Ministry of Education of the Czech Republic and by the European Commission within the Framework Program through I3-EURONS (Contract RII3-CT-2004-506065) and I3ENSAR (Project 262010).

Note added in proofs: After the acceptance of the paper the authors became aware of the publication of new results regarding the effects of scattering limiting the attainable precision in nuclear beta decay measurements with implanted sources [57-59], and of the production of intense ${ }^{31-35} \mathrm{Ar}$ beams with a nanostructured $\mathrm{CaO}$ target [60].

Open Access This is an open access article distributed under the terms of the Creative Commons Attribution License (http://creativecommons.org/licenses/by/4.0), which permits unrestricted use, distribution, and reproduction in any medium, provided the original work is properly cited.

\section{References}

1. ATLAS Collaboration (G. Aad et al.), Phys. Lett. B 716, 1 (2012).

2. CMS Collaboration (S. Chatrchyan et al.), Phys. Lett. B 716, 30 (2012).

3. Particle Data Group Collaboration (K.A. Olive et al.), Chin. Phys. C 38, 090001 (2014).

4. T.D. Lee, C.N. Yang, Phys. Rev. 104, 254 (1956). 
5. J.D. Jackson, S.B. Treiman, H.W. Wyld jr., Phys. Rev. 106, 517 (1957).

6. N. Severijns, M. Beck, O. Naviliat-Cuncic, Rev. Mod. Phys. 78, 991 (2006).

7. O. Naviliat-Cuncic, M. González-Alonso, Ann. Phys. (Berlin) 525, 600 (2013).

8. V. Cirigliano, S. Gardner, B. Holstein, Prog. Part. Nucl. Phys. 71, 93 (2013).

9. B.R. Holstein, J. Phys. G: Nucl. Part. Phys. 41, 114001 (2014).

10. K.K. Vos, H.W. Wilschut, R.G.E. Timmermans, Rev. Mod. Phys. 87, 1483 (2015).

11. A. Gorelov et al., Phys. Rev. Lett. 94, 142501 (2005).

12. D. Melconian et al., Phys. Lett. B 649, 370 (2007).

13. P.A. Vetter et al., Phys. Rev. C 77, 035502 (2008).

14. J. Pitcairn et al., Phys. Rev. C 79, 015501 (2009).

15. X. Fléchard et al., J. Phys. G: Nucl. Part. Phys. 38, 055101 (2011).

16. J.A. Behr, A. Gorelov, J. Phys. G: Nucl. Part. Phys. 41 114005 (2014)

17. E. Liénard et al., Hyperfine Interact. 236, 1 (2015).

18. M.G. Sternberg et al., Phys. Rev. Lett. 115, 182501 (2015).

19. B. Delauré et al., Hyperfine Interact. 146, 91 (2003).

20. M. Beck et al., Nucl. Instrum. Methods Phys. Res. A 503 , 567 (2003).

21. V.Yu. Kozlov et al., Phys. At. Nucl. 67, 1112 (2004).

22. V.Yu. Kozlov et al., Hyperfine Interact. 172, 15 (2006).

23. V.Yu. Kozlov et al., Nucl. Instrum. Methods Phys. Res. B 266, 4515 (2008).

24. M. Beck et al., Eur. Phys. J. A 47, 45 (2011).

25. S. Van Gorp et al., Phys. Rev. C 90, 025502 (2014).

26. E. Kugler, Hyperfine Interact. 129, 23 (2000).

27. S. Coeck et al., Nucl. Instrum. Methods Phys. Res. A 557, 516 (2006).

28. S. Coeck et al., Nucl. Instrum. Methods Phys. Res. A 572, 585 (2007).

29. E. Traykov et al., Nucl. Instrum. Methods Phys. Res. A 648, 1 (2011).

30. S. Van Gorp et al., Nucl. Instrum. Methods Phys. Res. A 638, 192 (2011)

31. M. Tandecki et al., Nucl. Instrum. Methods Phys. Res. A 629, 396 (2011).

32. N. Severijns et al., Phys. Rev. C 78, 055501 (2008).

33. L. Penescu et al., Rev. Sci. Instrum. 81, 02 A906 (2010).

34. F. Ames et al., Nucl. Instrum. Methods Phys. Res. A 538, 17 (2005).
35. L.S. Brown, G. Gabrielse, Rev. Mod. Phys. 58, 233 (1986).

36. T. Porobić et al., Nucl. Instrum. Methods Phys. Res. A 785, 153 (2015).

37. V.M. Lobashev, P.E. Spivak, Nucl. Instrum. Methods Phys. Res. A 240, 305 (1985).

38. A. Picard et al., Nucl. Instrum. Methods Phys. Res. B 63 , 345 (1992)

39. L. Bornschein, Nucl. Phys. A 752, 14 (2005).

40. M. Simson et al., Nucl. Instrum. Methods Phys. Res. A 611, 203 (2009).

41. FASTER Data Acquisition System, LPC Caen, http:// faster.in2p3.fr.

42. C. Fontbonne, FASTER Manual (2012), http://faster. in 2 p $3 . f r /$ index . php/documentation?download=16: faster-qdc-tdc-module-user-manual.

43. R. Brun, F. Rademakers, Nucl. Instrum. Methods Phys. Res. A 389, 81 (1997) http://root.cern.ch/.

44. E. Liénard et al., Nucl. Instrum. Methods Phys. Res. A 551, 375 (2005).

45. W.H. Press et al., Numerical Recipes in $C$, 2nd edition (Cambridge University Press, 2002).

46. A.T. Laffoley et al., Phys. Rev. C 92, 025502 (2015).

47. J. Ziegler, SRIM - The Stopping and Range of Ions in Matter (2015) http://www.srim.org.

48. D. Zákoucký, internal report (2014).

49. M. Wang et al., Chin. Phys. C 36, 1603 (2012).

50. M. Tandecki, PhD Thesis, Katholieke Universiteit Leuven (2011), http://fys.kuleuven.be/iks/wi/files/ final-version-thesis-michael-tandecki.pdf.

51. P. Friedag, PhD Thesis, Westfälischen WilhelmsUniversität Münster (2013) http://repositorium.unimuenster.de/document/miami/627d5ad1-6043-417b94ba-5c87309bc2bd/diss_friedag.pdf.

52. F. Glück et al., Prog. Electromagn. Res. B 32, 319 (2011)

53. C. Couratin et al., Phys. Rev. A 88, 041403(R) (2013).

54. E.S. Parilis, L.M. Kishinevskii, Sov. Phys. - Solid State 215, 885 (1960).

55. F. Glück, The Penning discharge, internal KATRIN document (2007).

56. R. Maisonobe, PhD Thesis, Institut Laue-Langevin de Grenoble (2014).

57. F. Wauters et al.., Phys. Rev. C 80, 062501(R) (2009).

58. F. Wauters et al.., Phys. Rev. C 82, 055502 (2010).

59. G. Soti et al., Phys. Rev. C 90, 035502 (2014).

60. J.P. Ramos et al., Nucl. Instrum. Methods Phys. Res. B 320, 83 (2014). 